\title{
Loading Dependence of the Diffusion Coefficient of Methane in Nanoporous Materials
}

\author{
E. Beerdsen, ${ }^{*}, \dagger$ D. Dubbeldam, ${ }^{\dagger}$ and B. Smit ${ }^{\dagger}, \hbar$ \\ Department of Chemical Engineering, University of Amsterdam, Nieuwe Achtergracht 166, \\ 1018 WV Amsterdam, The Netherlands, and Centre Européen de Calcul Atomique et Moléculaire (CECAM), \\ Ecole Normale Supérieure 46, Allée d'Italie, 69007 Lyon, France
}

Received: June 30, 2006; In Final Form: August 28, 2006

\begin{abstract}
In this work, we use molecular simulations to study the loading dependence of the self-and collective diffusion coefficients of methane in various zeolite structures. To arrive at a microscopic interpretation of the loading dependence, we interpret the diffusion behavior in terms of hopping rates over a free-energy barrier. These free-energy barriers are computed directly from a molecular simulation. We show that these free-energy profiles are a convenient starting point to explain a particular loading dependence of the diffusion coefficient. On the basis of these observations, we present a classification of zeolite structures for the diffusion of methane as a function of loading: three-dimensional cagelike structures, one-dimensional channels, and intersecting channels. Structures in each of these classes have their loading dependence of the free-energy profiles in common. An important conclusion of this work is that diffusion in nanoporous materials can never be described by one single effect so that we need to distinguish different loading regimes to describe the diffusion over the entire loading range.
\end{abstract}

\section{Introduction}

Membranes function because of differences in diffusion and adsorption of the molecules that are adsorbed in these materials. Many different materials are used as membranes. Lipid bilayers in cell membranes and molecular sieves such as zeolites in industrial separation are just a few examples. These nanoporous materials contain pores that have sizes similar to the dimensions of the adsorbed molecules and therefore impose a tight confinement. This makes the diffusion behavior of adsorbed molecules in these materials very different from diffusion in a bulk fluid. ${ }^{1-10}$

Although these systems are well-studied, their diffusion properties remain poorly understood. An intensive research effort to measure diffusion rates in nanoporous materials augmented the possibilities of determining diffusion rates in nanoporous materials, such as zeolites, metal-organic frameworks, ion channels, etc. However, while it is often possible to determine the diffusion as a function of adsorbate loading rather accurately, a proper understanding of diffusion behavior is still lacking. For a given structure, it is usually impossible to predict whether the diffusion will decrease, increase, or remain constant when the loading is increased.

Molecular dynamics simulations have often been used to obtain the diffusion coefficients. ${ }^{1,2,11-18}$ However, the results of a molecular dynamics, diffusion coefficients as a function of loading or temperature, are equally difficult to rationalize at the molecular level as the results of experiments, whereas an important part of diffusion research is to relate the observed trends in the diffusion coefficients to the behavior of the molecules at the molecular level. Some groups have used transition-state theory, to gain insight in the diffusion properties in zeolites. ${ }^{3,19-28}$ However, these studies were often limited to

* Corresponding author e-mail: beerdsen@science.uva.nl.

$\dagger$ University of Amsterdam.

$\doteqdot$ Centre Européen de Calcul Atomique et Moléculaire (CECAM). the infinite dilution limit, whereas most experimental values were obtained at nonzero loading. ${ }^{29-31}$ Only recently, a dynamically corrected transition-state theory method (dcTST) has been developed that can be applied to study diffusion in confined systems beyond the infinite dilution limit. ${ }^{32}$ This method can provide a molecular explanation for diffusion behavior in terms of free energy. ${ }^{33,34}$ Armed with this method, we can now take a more generalized look on diffusion in confined systems.

In this paper, we combine molecular dynamics simulations with calculations using the new dcTST method to gain insight in the diffusion of methane in twelve different types of zeolites. We calculate self and corrected diffusivities as a function of loading and find an explanation by studying the free-energy changes as the loading is increased. Following the work of Skoulidas and Sholl, ${ }^{1}$ who published MD results for four different zeolite structures, we analyze a representative set of twelve zeolite structures for this study. We can divide the twelve zeolites in four different groups, that each have their own diffusion behavior. We have chosen methane for this study because it simplifies the interpretation of free-energy profiles and the explanation of our results. But the method is by no means limited to methane. A similar study could be made for any other molecule.

The remainder of the paper is organized as follows. We begin in the next section with diffusion theory, the Darken approximation, lattice approaches, and the Reed-Ehrlich model. Section III summarizes the methods used, molecular dynamics, and dynamically corrected transition state theory; section IV introduces the zeolites used for this study. The diffusion results for the four zeolite groups are presented in section V. In section VI, we evaluate the results and compare them to the ReedEhrlich method, and section VIII contains the conclusions.

\section{Diffusion Theory}

Diffusion can be expressed by a diffusion coefficient in several ways. In practical experiments, such as measurements 
of the uptake and permeation rate, the diffusion measured is usually the transport diffusion coefficient, $D_{\mathrm{t}}$. This coefficient is defined by Fick's law

$$
J=-D(c)_{\mathrm{t}} \nabla c
$$

where $J$ is the sorbate flux when a concentration gradient, $\nabla c$, is applied.

To obtain a diffusion coefficient that is presumably less dependent on loading, $D_{t}$ is often converted to the corrected diffusion, $D_{\text {c }}$. This is also known as the Maxwell-Stefan or Darken diffusion coefficient and can be obtained from

$$
D_{\mathrm{t}}(\theta)=D_{\mathrm{c}} \frac{\partial \ln f}{\partial \ln q}=D_{\mathrm{c}} \times \gamma(\theta)
$$

where $q$ is the loading in the sorbent, $\theta$ the fractional occupancy, and $f$ the fugacity. ${ }^{4}$ The corrected diffusivity is the collective diffusion behavior of all adsorbate particles, including interparticle correlations and can be interpreted as the movement of the center of mass of all particles together

$$
\begin{aligned}
D_{\mathrm{c}} & =\lim _{t \rightarrow \infty} \frac{1}{6 N t} \sum_{i=1}^{N} \sum_{j=1}^{N} r_{i}(0) r_{j}(t) \\
& =\lim _{t \rightarrow \infty} \frac{1}{6 N t}\left(\sum_{i=1}^{N} \Delta r_{i}(t)\right)^{2}
\end{aligned}
$$

One other common measure of diffusion is the self-diffusion coefficient, $D_{\mathrm{s}}$. It is the diffusion of a single tagged particle moving around in a sea of other particles. It is defined as

$$
D_{\mathrm{s}}=\lim _{t \rightarrow \infty} \frac{1}{6 N} \sum_{i=1}^{N} \Delta r_{i}(t)^{2}
$$

where $\Delta r_{i}$ is the displacement of particle $i$ at time $t$ with respect to time 0 and $N$ is the number of particles. This is the diffusion coefficient that can be obtained by microscopic methods, such as pulsed-field gradient NMR (PFG-NMR).

In general, the corrected diffusion is higher than the self diffusion because the corrected diffusion contains interparticle correlations, which have a positive contribution or, viewed differently, the self diffusion is lowered by single-particle backcorrelations (the increased probability of a particle jumping back to its previous position because this position has a higher probability of being empty).

A. Darken Approximation. At the infinite dilution limit, $D_{\mathrm{s}}$ and $D_{\mathrm{c}}$ are strictly equivalent. This observation has often been used to approximate eq 2 by replacing $D_{\mathrm{c}}$ with $D_{\mathrm{s}}$. This is called the Darken approximation, and eq 2, under these conditions, is called the Darken equation. ${ }^{5}$ For reasons of convenience, the corrected diffusivity has been assumed to be relatively insensitive to changes in concentration, thus making it possible to use the Darken equation at arbitrary loading. It has for instance been used to relate macroscopic and microscopic diffusion processes. Although many deviations have been found, where the corrected diffusivity was concentration dependent, they were seen as exceptions to the general rule. For methane in MFI-type zeolite, support for the concentration independence was found almost up to high, but not maximum loading. ${ }^{1,33}$

B. Lattice Models and Correlations. Diffusion is often studied by considering particles' movements as a hopping process on a lattice. When the hopping rates between the different lattice points are known, the diffusion can be computed by using the formula

$$
D_{\mathrm{s}}=\frac{1}{2 d} k \lambda^{2}
$$

where $D_{\mathrm{s}}$ is the self-diffusion coefficient, $d$ the dimensionality of the system, and $k$ the hopping rate from a lattice site to any of its neighboring lattice sites. It is often possible to coarsegrain a system for computation on a lattice, but care should be taken when diffusion coefficients are calculated in this way. Particles diffusing in a "real" system might be inclined to hop onward in the same direction (so-called multijumps) or back in the direction from which they came (vacancy correlations). Particles that jump to a new position can attract other particles to jump after them. To obtain a correct diffusion coefficient, all these effects should be taken into account. For a more elaborate discussion on interparticle and time correlations, we refer to the paper of Ala-Nissila et al. ${ }^{35}$

C. Diffusion Regimes and the Reed-Ehrlich Model. The starting point for our explanation of diffusion on a molecular scale is the Reed-Ehrlich model, which is often used to describe diffusion phenomena. ${ }^{36}$ In this model, diffusion in a nanoporous material is considered as a hopping process on a lattice of adsorption sites, in which all sites have equal adsorption energies. The only restriction is that a particle cannot move to a site that is occupied by another particle. In such a system, the corrected diffusion decreases linearly with loading

$$
D_{\mathrm{c}}(q)=D_{\mathrm{c}}(0) \times \frac{\left(q_{\max }-q\right)}{q_{\max }}=D_{\mathrm{c}}(0) \times(1-\theta)
$$

where $q$ is the loading, $D_{\mathrm{c}}(0)$ is the corrected diffusion at infinite dilution, $q_{\max }$ is the maximum loading, and $\theta$ is the fractional loading or occupancy.

The Reed-Ehrlich method was originally introduced for surface diffusion, but the model has recently been transferred to zeolites by Krishna and co-workers ${ }^{37}$ and has been used successfully in several studies. .,38-40 $^{2}$

The model works well for materials in which all adsorption sites are equal (in other words, diffusional barriers do not change as a function of loading or $f=1$ in the Reed-Ehrlich formulation) and for which the adsorption can thus be described by a single Langmuir isotherm.

However, most nanoporous materials have several types of adsorption sites, mutually differing in adsorption energy, and adsorption in such materials is described by an $n$-site Langmuir isotherm. For this type of material, eq 7 cannot be used, and we have to use eq 8 instead

$$
D_{\mathrm{c}}(q)=D_{\mathrm{c}}(0) \times \frac{\partial \ln q}{\partial \ln f}=D_{c}(0) \times \frac{1}{\gamma}
$$

where $f$ is the adsorbate fugacity and $1 / \gamma$ gives the fraction of empty sites as a function of loading for an $n$-site Langmuir isotherm. ${ }^{37,41-43}$ For a single Langmuir isotherm, eq 8 reduces to eq 7. The isotherms and $1 / \gamma$ as a function of loading for a single-site and dual-site system are shown in Figure 1.

Diffusion in systems for which the energy of the different adsorption sites is not influenced by loading (for example, because there are no specific adsorption sites or the sites are very far apart) can be very well described by eq 8 . It is in the nature of a Langmuir isotherm that $1 / \gamma$ is never constant over loading. This leaves us with two possible diffusion regimes:

A1. As the structure fills up, the diffusion decreases with loading. As adsorption sites are being filled up, less space is available for molecules to move around. For single-Langmuir systems, we see a linear decrease of diffusion as a function of 

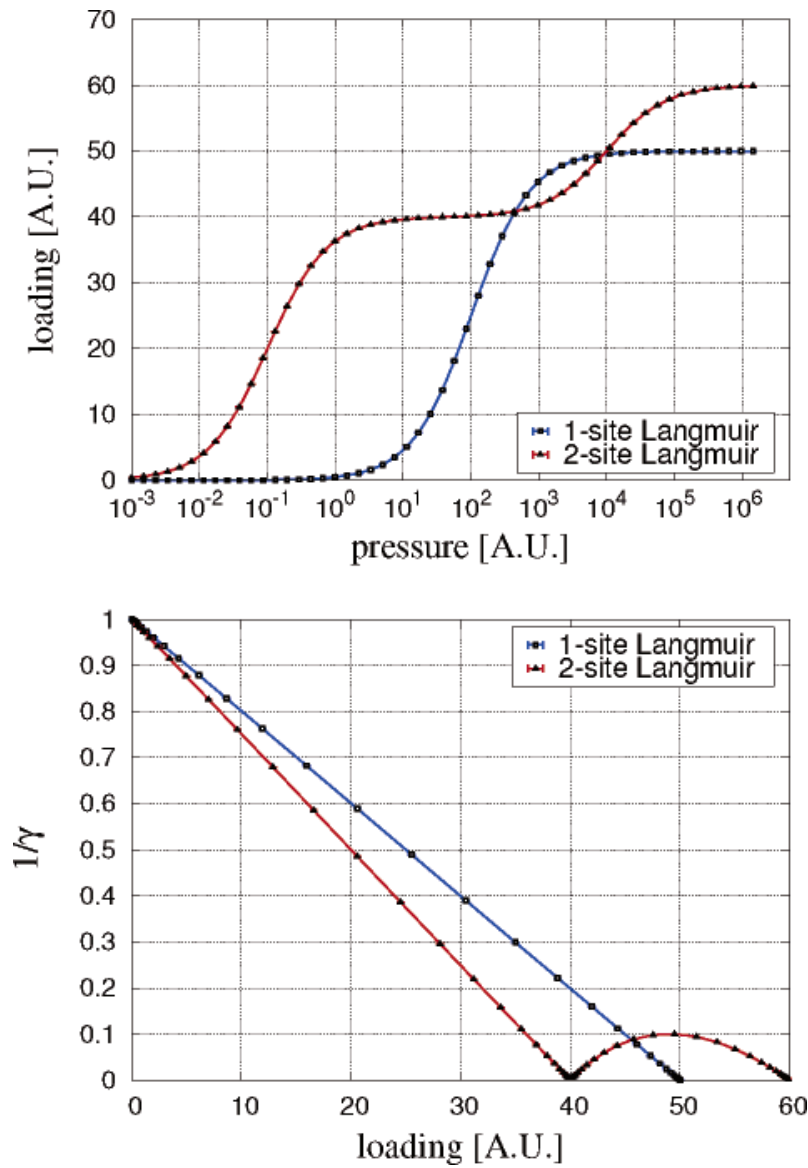

Figure 1. Isotherm and $1 / \gamma$ for a single-site Langmuir and a dual-site Langmuir system. Loading and pressures are given in arbitrary units.

loading (see Figure 1 (bottom)). For multiple-site Langmuir systems, a decrease in the adsorption is only observed in part of the loading range: the parts where the derivative of the adsorption isotherm decreases, or in other words, where the number of available adsorption sites diminishes. This decrease can be linear (as in Figure 1 (bottom) for a dual-site Langmuir isotherm, up to a loading of 40), but it can also have a more complex form. In any nanoporous system, this behavior is observed at very high loadings. When the available space is almost filled up completely, the molecules move ever more slowly, until their movement comes to a complete halt and the diffusion plunges to zero. The loading at which this happens defines the maximum loading of the material.

A2. At an inflection in the adsorption isotherm, the number of effective adsorption sites increases; the diffusion increases with loading. In systems with multiple-Langmuir adsorption behavior, not all adsorption sites are filled at the same time. At a certain loading, new adsorption sites become available, or a reordering of the adsorbed molecules takes place. This is observed as an inflection in the adsorption isotherm and, correspondingly, an increase in the diffusion.

Most system's diffusion behavior cannot be captured completely by the adsorption isotherm. It is not uncommon for the energy of adsorption sites to be dependent on whether the neighboring adsorption sites are occupied. In the Reed-Ehrlich model, this change in the free energy can be captured by including a parameter $f$ that is dependent on the loading. ${ }^{37,39,40}$ This, however, requires a reasonably detailed prior knowledge about the system under study. We can calculate free-energy profiles of periodic nanoporous structures by plotting the free energy as a function of the position. These profiles can change significantly over loading, following the energy changes of the adsorption sites. This gives rise to two additional diffusion regimes:

B1. Free-energy barriers decrease; the diffusion increases with loading. Favorable interaction of particles with the "wall" of the nanoporous material is replaced by less favorable interactions with neighboring adsorbed particles. This increases the free energy of adsorption regions and decreases the net free-energy barriers that have to be overcome to move to a next adsorption region. A decrease in the effective free-energy barrier accelerates the diffusion. This is also the underlying cause of the so-called window effect. $27,28,44-47$

B2. Free-energy barriers increase; the diffusion decreases with loading. Likewise, it is possible that the addition of extra molecules causes an increase in the free-energy barriers that molecules must overcome to move around. For example, this could be the case for polar molecules, for which the interaction with other adsorbed molecules is more favorable than the interaction with the wall of the porous material. In such systems, when the loading is increased, the adsorbed molecules will stick more and more to each other and to their preferred positions, causing a decrease in the diffusion. A similar behavior is observed for any molecule in any nanoporous material, at very high loadings, when the structure is almost completely full. The molecules inside the material are packed tightly, and movement from one position to the next involves crossing increasingly high free-energy barriers.

In real systems, diffusion is a complex interplay of all four effects. To demonstrate this, in this paper we will turn our attention to diffusion in zeolites.

\section{Methods}

The zeolites were modeled as rigid frameworks for which the interactions with the alkane molecules are dominated by the oxygen atoms. ${ }^{48}$ For the guest molecules, a united-atom model was used, ${ }^{49}$ in which we consider the $\mathrm{CH}_{4}$ group as a single reaction center with its own effective potential. The potential parameters are optimized to reproduce adsorption properties in pure silica confinements. ${ }^{50,51}$

A. Molecular Dynamics. The diffusion was calculated using molecular dynamics (MD) calculations. In an MD calculation, Newton's equations are being solved to study particle positions as a function of time and thus obtain a mean square displacement as a function of time. This mean square displacement can easily be converted into a self-diffusion coefficient with

$$
D_{\mathrm{s}, x}=\lim _{t \rightarrow \infty} \frac{1}{2 N t} \sum_{i=1}^{N}\left(\Delta x_{i}(t)\right)^{2}
$$

and subsequently

$$
D_{\mathrm{s}}=\frac{D_{\mathrm{s}, x}+D_{\mathrm{s}, y}+D_{\mathrm{s}, z}}{3}
$$

In a similar fashion, the corrected diffusivity can be obtained by calculating the mean square displacement of the center of mass

$$
D_{\mathrm{c}, x}=\lim _{t \rightarrow \infty} \frac{1}{2 N t}<\left(\sum_{i=1}^{N} r_{i, x}(t)\right)^{2}>
$$

and subsequently 

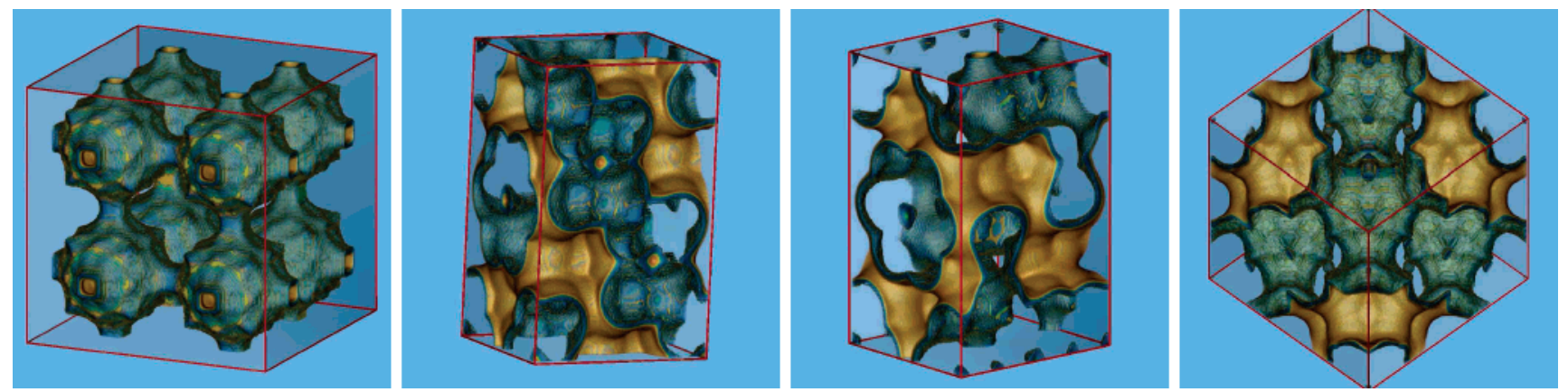

Figure 2. Structure of the unit cells of four cage-type zeolites (LTA, CHA, ERI, and FAU).

$$
D_{\mathrm{c}}=\frac{D_{\mathrm{c}, x}+D_{\mathrm{c}, y}+D_{\mathrm{c}, z}}{3}
$$

B. Dynamically Corrected Transition State Theory. Alternatively, self-diffusion coefficients can be computed using a dynamically corrected transition state theory method. In addition to diffusion coefficients, such a method can yield an explanation of the diffusion behavior in terms of free-energy differences. Transition state theory regards diffusion as a hopping process on a lattice, where the hopping from some state A to another state B is limited by a free-energy barrier between the two states. When the hopping rates between the different lattice points are known, the self-diffusion coefficient can be obtained using the formula

$$
D_{\mathrm{s}}=k_{\mathrm{A} \rightarrow \mathrm{B}} \lambda^{2}=\frac{1}{6} k \lambda^{2}
$$

where $D_{\mathrm{S}}$ is the self-diffusion coefficient, $k_{\mathrm{A} \rightarrow \mathrm{B}}$ is the hopping rate from $\mathrm{A}$ to $\mathrm{B}, \lambda$ is the lattice distance (i.e., the distance between states $A$ and $B$ ), and $k$ is the hopping rate from $\mathrm{A}$ to any of its neighboring lattice sites. Equation 13 gives the conversion from hopping rates to diffusion coefficients for a cubic lattice, but a similar equation can be derived for any other lattice topology.

When diffusion is considered to be a hopping process on a lattice, it is convenient to split the hopping rate $k$ into two parts

$$
k=k^{\mathrm{TST}} \times \kappa,
$$

where $k^{\mathrm{TST}}$ is the trial hopping rate, determined from transition state theory (i.e., the frequency with which a particle attempts to jump to a neighboring lattice site) and $\kappa$ is the dynamical correction factor or, in other words, the probability that the particle will be accepted at the next lattice point. In this paper, we will make use of this concept because it allows us to split the diffusion into a free-energy contribution, arising from the structure of the confinement and the ordering of the adsorbates inside, contained in $k^{\mathrm{TST}}$, and an interparticle collision contribution, $\kappa$.

In the Bennett-Chandler approach, ${ }^{52-54}$ one computes the hopping rate $k_{\mathrm{A} \rightarrow \mathrm{B}}$ over the barrier in two steps. First, the relative probability $P\left(q^{*}\right)$ is computed to find a particle on top of the barrier, given that it is in state $\mathrm{A}$, and subsequently, the averaged velocity at the top of the barrier $\sqrt{k_{\mathrm{B}} T / 2 \pi m}$ (assuming that the particle velocities follow a Maxwell-Boltzmann distribution) and the probability, $\kappa$, that the system ends up in state $\mathrm{B}$. The transmission rate $k_{\mathrm{A} \rightarrow \mathrm{B}}$ from cage $\mathrm{A}$ to cage $\mathrm{B}$ is then given by

$$
\begin{gathered}
k_{\mathrm{A} \rightarrow \mathrm{B}}=\kappa \times \sqrt{\frac{k_{\mathrm{B}} T}{2 \pi m}} \times P\left(q^{*}\right) \\
P\left(q^{*}\right)=\frac{\mathrm{e}^{-\beta F\left(q^{*}\right)}}{\int_{\text {cage }} \mathrm{e}^{-\beta F(q)} \mathrm{d} q}
\end{gathered}
$$

where $\beta=1 /\left(k_{\mathrm{B}} T\right), k_{\mathrm{B}}$ is the Boltzmann constant, $T$ is the temperature, $m$ is the mass involved in the reaction coordinate, and $F(q)$ is the free energy as a function of $q$. In first-order approximation, TST assumes that all particles that reach the barrier with a velocity toward B do eventually end up in B (i.e., $\kappa=1$ ). For soft-potential lattice models at nonzero loading, $\kappa$ will be smaller than 1 .

We can choose the reaction coordinate $q$ as the position of one of the atoms of the diffusing molecules. ${ }^{27}$ In dynamically corrected TST (dcTST), the transmission coefficient, $\kappa$, corrects for recrossing events (i.e., it corrects for trajectories which cross the transition state from A but fail to end up in B). The return of particles to cage A can be attributed to various causes. If the reaction coordinate is chosen in a nonoptimal way, this choice of order parameter underestimates the free energy of the true transition state, but the dynamic correction $\kappa$ is the exact correction compensating for our choice of reaction coordinate..$^{53}$ Furthermore, $\kappa$ can be smaller than one, because of interparticle collisions: particles coming off the barrier and colliding with other particles before reaching equilibrium in state $B$, thereby returning to state $A$. If one manages to find a "perfect" reaction coordinate (i.e., $\kappa=1$ at zero loading), one can regard $\kappa$ as a correlation and collision frequency parameter: the lower the value of $\kappa$, the higher the number of collisions. In such cases it is possible to consider diffusion as a product of two contributions

$$
\begin{aligned}
D_{S} & =k_{\mathrm{A} \rightarrow \mathrm{B}} \times \lambda^{2}=\kappa \times \sqrt{\frac{k_{\mathrm{B}} T}{2 \pi m}} \times P\left(q^{*}\right) \times \lambda^{2} \\
& =\kappa \times D_{\mathrm{S}}^{\mathrm{TST}}
\end{aligned}
$$

In this equation $D_{\mathrm{S}}^{\mathrm{TST}}$ is the free-energy contribution to the self diffusion, the part of the diffusion that is governed by free energy barriers: effects of the topology of the confinement and the changes in the effective topology as a function of loading. $\kappa$ is the contribution to the diffusion of interparticle collisions, which in general have a lowering effect on the diffusion. For more details about the dcTST method, we refer the reader to ref 35 .

By calculating diffusion in this way, we can distinguish between topology contributions (included in $D_{\mathrm{S}}^{\mathrm{TST}}$ ) and particle collision contributions (included in $\kappa$ ). This leads to a better understanding of diffusion behavior as a function of loading, as we will show in the Results section. 
TABLE 1: Data of Four Cage-Type Zeolites (LTA, ERI, CHA, and FAU), Four Channel-Type Zeolites (SAS, LTL, MTW, and AFI), and Four Intersecting Channel-Type Zeolites (BOG, BEC, ISV, and MFI $)^{a}$

\begin{tabular}{|c|c|c|c|c|c|c|c|c|c|}
\hline & \multirow{2}{*}{$\begin{array}{l}\text { ring } \\
\text { size }\end{array}$} & \multirow{2}{*}{$\begin{array}{c}\text { window } \\
\text { diameter }(\AA)\end{array}$} & \multirow{2}{*}{$\begin{array}{c}\text { cage } \\
\text { diameter }(\AA)\end{array}$} & \multirow[b]{2}{*}{$R_{\mathrm{ctw}}$} & \multicolumn{3}{|c|}{ unit cell dimensions $(\AA)$} & \multirow[b]{2}{*}{ unit-cell type } & \multirow[b]{2}{*}{ simulation box } \\
\hline & & & & & $a$ & $b$ & $c$ & & \\
\hline LTA & 8 & 4.1 & 10 & 2.44 & 24.555 & 24.555 & 24.555 & cubic & $2 \times 2 \times 2$ \\
\hline ERI & 8 & $3.6-5.1$ & 11 & 3.06 & 22.953 & 13.252 & 14.810 & orthorhombic & $2 \times 3 \times 3$ \\
\hline CHA & 8 & 3.8 & 8.5 & 2.24 & 15.075 & 23.907 & 13.803 & orthorhombic & $2 \times 2 \times 3$ \\
\hline FAU & 12 & 7.4 & 9 & 1.22 & 25.028 & 25.028 & 25.028 & cubic & $1 \times 1 \times 1$ \\
\hline SAS & 8 & 4.2 & 10 & 2.38 & 14.322 & 14.322 & 10.424 & tetragonal & $2 \times 2 \times 3$ \\
\hline LTL & 12 & 7.1 & 13 & 1.83 & 31.984 & 18.466 & 7.476 & orthorhombic & $1 \times 2 \times 4$ \\
\hline MTW & 12 & $5.6-6.0$ & 8 & 1.42 & 24.863 & 5.012 & 24.326 & monoclinic, $\beta=107.722^{\circ}$ & $1 \times 16 \times 1$ \\
\hline AFI & 12 & 7.3 & 10 & 1.37 & 23.774 & 13.726 & 8.484 & orthorhombic & $2 \times 2 \times 8$ \\
\hline BOG & $12 / 10$ & $7.0 / 5.5-5.8$ & $b$ & - & 20.236 & 23.798 & 12.798 & orthorhombic & $2 \times 2 \times 3$ \\
\hline $\mathrm{BEC}$ & $12 / 12$ & $6.6-7.7 / 5.6$ & $b$ & - & 13.100 & 13.100 & 13.800 & tetragonal & $3 \times 3 \times 3$ \\
\hline ISV & $12 / 12$ & $6.1-6.5 / 5.9-6.6$ & $b$ & - & 12.853 & 12.853 & 25.214 & tetragonal & $2 \times 2 \times 1$ \\
\hline MFI & $10 / 10$ & $5.1-5.5 / 5.3-5.6$ & $b$ & - & 20.022 & 19.899 & 13.383 & orthorhombic & $2 \times 2 \times 4$ \\
\hline
\end{tabular}

${ }^{a}$ For each zeolite type, the table lists the window ring size in number of oxygen atoms per ring, the window diameter and the cage diameter $(\AA)$, the cage-to-window ratio, $R_{\mathrm{ctw}}$, the unit cell dimensions in the three directions ( $\AA$ ), the unit cell form, and the size of the simulation box (the number of unit cells in the $x, y$, and $z$ directions). The cage and window data (left and right) for intersecting channel-type zeolites are the values for the channels in the different directions. Where the window diameter is given as a range (e.g., 3.6-5.1 for the ERI-type zeolite), this signifies that the windows have an oval shape. The values for $R_{\text {wtc }}$ in these cases are calculated as the ratio of the smallest diameter of the oval to the diameter of the cage. ${ }^{b}$ For the intersecting channel-type zeolites, it is not practical to assign cage widths and window-to-cage ratios for reasons given in the text.

C. Computational Details. We used the Verlet integration algorithm with a time step of $0.5 \mathrm{fs}$ and, depending on the diffusion speed, a total simulation time of between 1.5 and 1000 ns, so that the error bars were smaller than the symbol size. The NVT ensemble was imposed using a Nosé-Hoover thermostat. Free-energy profiles were obtained from Monte Carlo simulations using the "histogram method" described in ref 32. The simulation box sizes for the various simulated systems are given in Table 1.

\section{Zeolite Structures}

Zeolites are nanoporous solids, very suitable for and used frequently as model systems for understanding molecular diffusivity in porous media, because of their well-defined crystalline structure with highly ordered, periodic confinements. ${ }^{1,2,5,6,24,55,56}$

Zeolites exist in a wide variety of structures. Currently, over 130 different topologies are known. ${ }^{57}$ These structures are commonly divided in three types. Channel-type zeolites consist of more or less linear channels that run in one direction only. Therefore, diffusion in such structures occurs in one dimension only. Intersecting channel-type zeolites consist of channels in different directions, that cross each other at so-called intersections. Three-dimensional cage-type zeolites consist of cages, connected by narrow windows.

We analyze a representative set of zeolite structures. We have tried to make our set of zeolites as broad as possible, by choosing twelve different, widely varying topologies. We calculated loading-dependent diffusion in four different cagetype zeolites (LTA, ERI, CHA, and FAU), four channel-type zeolites (AFI, MTW, LTL, and SAS) and four intersecting channel-type zeolites (MFI, BOG, BEC, and ISV). In this section, we give a short overview of the different topologies. The structure coordinates for the twelve zeolites were taken from refs $58-69$.

A. Three-Dimensional Cage-Type Zeolites. Figure 2 shows the structures of the cage-type zeolites we used in this study. In Table 1 we summarize some geometric data on these four structures. For each zeolite, the table lists the window ring size in number of oxygen atoms per ring, the window diameter and the cage diameter, the cage-to-window ratio, $R_{\mathrm{ctw}}$, which is defined as $R_{\text {ctw }}=$ cage diameter/window diameter, the unit- cell dimensions, the unit-cell form, and the size of the simulation box. $R_{\mathrm{ctw}}$ can be used as a measure of confinement by the cagetype structure; the larger the value of $R_{c t w}$, the larger the difference between the cage width and the window diameter and the larger the free-energy barrier at the window is expected to be.

Of the four cage-type zeolites, erionite-type (ERI-type) zeolite has the largest $R_{\mathrm{ctw}}$. ERI-type zeolites have long cages, elongated in the $c$ direction, that are each connected to six other cages, three on each side of the long cages. The windows connecting these cages are elliptical, their diameter varies from $3.6 \AA$ in the $b$ direction to $5.1 \AA$ in the $c$ direction. In our simulations, we used a rectangular version of the unit cell, where one unit cell of erionite contains 4 cages.

Zeolite types LTA and CHA have comparable values for $R_{\mathrm{ctw}}$, 2.43 and 2.24 respectively, smaller than ERI-type zeolite. The structure of LTA-type zeolite consists of almost spherical cages of about $10 \AA$ in diameter, connected by narrow windows of about $4 \AA$ in diameter. One unit cell consists of 8 cubically arranged cages and the windows form entropic, not energetic barriers. Zeolite A, Linde Type-A (LTA), is a microporous crystalline material widely used in the detergent industry. Its supercage structure is useful in spatiospecific catalysis, typically of $n$-paraffins and olefins. One use is in paraffin cracking. The small entry pore is selective toward linear paraffins, and cracking can occur on sites within the supercage to produce smaller chain alkanes. ${ }^{70}$ Zeolite $\mathrm{A}$ is also widely used in in ion-exchange separation. ${ }^{71}$

We find a slightly smaller value for $R_{\text {ctw }}$ in chabazite-type (CHA) zeolite. It consists of slightly elongated cages that are each connected to six other cages in a near-cubic fashion: the angles $\alpha, \beta$, and $\gamma$ in the trigonal unit cell are all $94^{\circ}$. For our simulations, a rectangular unit cell was constructed. The CHAtype zeolite is industrially used in the formation of light olefins from methanol and in xylene isomerization.

The smallest value of $R_{\mathrm{ctw}}$ is that of faujasite-type (FAU) zeolite. This zeolite both has large cages and large windows. One unit cell of FAU-type zeolite contains eight cages with a shape similar to those in LTA-type zeolite, but arranged in a tetrahedral manner. Depending on the atomic composition, FAUtype zeolites include zeolites $\mathrm{X}$ and $\mathrm{Y}$. The most important use of zeolite $\mathrm{Y}$ is as a cracking catalyst. ${ }^{71-73}$ 

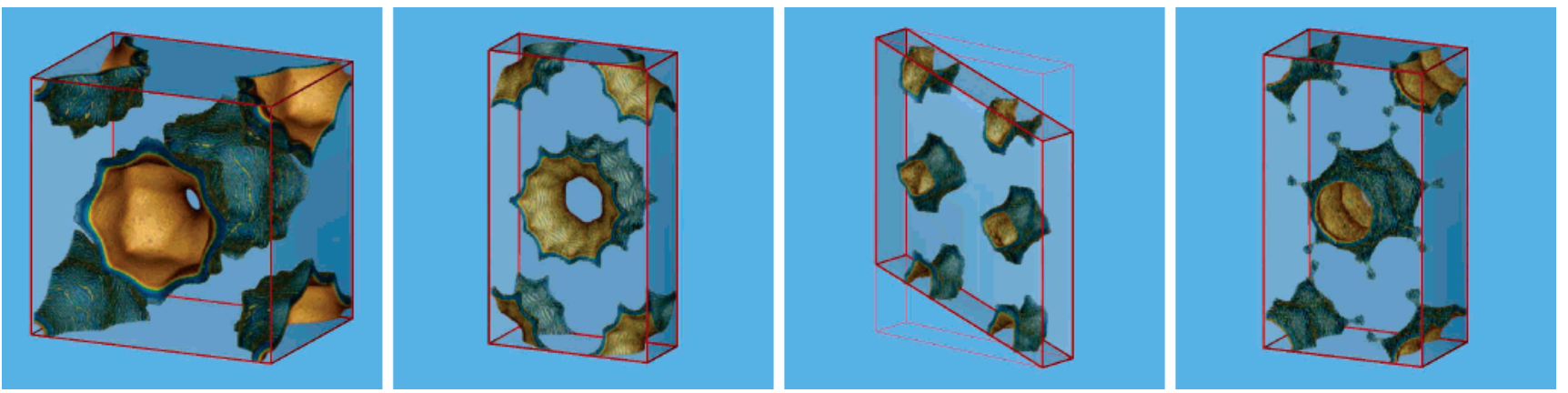

Figure 3. Structure of the unit cells of channel-type structures SAS, LTL, MTW, and AFI.
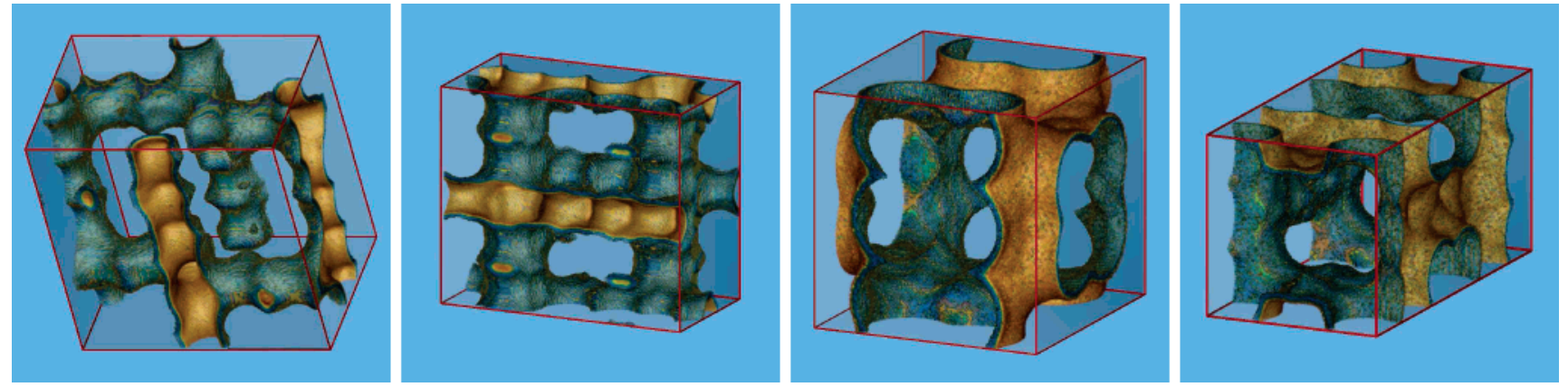

Figure 4. Structure of the unit cells of intersecting channel-type structures MFI, BOG, BEC, and ISV.

B. Channel-Type Zeolites. The class of channel-type zeolites is very diverse. All zeolites whose diffusion occurs in one direction only, in straight channels that are not interconnected, fall under this category. Thus, channel-type zeolites can consist of one-dimensionally connected cages, but they can also consist of very smooth straight tubes or channels with any degree of smoothness in between. Therefore, we cannot pick a single zeolite to represent the entire class of channel-type zeolites. To gain insight in the diffusion behavior in this class, we will therefore turn our attention to four different channel-type zeolites: AFI, MTW, LTL, and SAS. In this work, we only consider channels that are sufficiently big or molecules that are sufficiently small such that two molecules can pass each other. In these one-dimensional channels, one can observe an interesting case of geometry correlations if the molecules cannot pass each other. In such a system one does not observe diffusive behavior but single-file diffusion. Single-file diffusion has been extensively investigated in experiments ${ }^{74-77}$ and in simulations. ${ }^{78-83}$

Figure 3 shows the structures of these four zeolites. The accompanying geometric data are given in Table 1. For channeltype zeolites, the cage-to-window ratio, $R_{\mathrm{ctw}}$, can be used as a measure of "cagelikeness": the larger the value of $R_{c t w}$, the larger the difference in width between the widest parts and the narrowest parts of the channels, and the larger, again, the freeenergy barriers are expected to be.

One of the smoothest tubelike zeolites is AFI-type zeolite. It consists of straight channels that are not interconnected. Diffusion occurs in the $z$ direction only. As shown in Figure 3, a unit cell of AFI contains two channels, and the diameter of the channels varies between about 7.3 and $10 \AA$ (a $R_{\mathrm{ctw}}$ of 1.37 ). AFI-type zeolites are used in the so-called Fischer-Tropsch synthesis, for the production of clean fuels and chemical products from natural gas and coal. ${ }^{84,85}$ Other applications include the use in a zeolite-dye microlaser. ${ }^{86}$

Another relatively smooth channel-type zeolite is MTW-type zeolite. Like AFI, this zeolite consists of straight channels, but in the MTW-type zeolite, they range between 5.6 and $8 \AA$ in diameter (a $R_{\mathrm{ctw}}$ of 1.42) and run in the $y$ direction. MTW is among the smallest 12-membered ring zeolite structures. ${ }^{87} \mathrm{~A}$ commonly used zeolite with MTW-topology is ZSM-12. It is known for its exceptional time stability and is used in acidcatalyzed reactions. ${ }^{87}$

An intermediate channel-type zeolite is LTL-type zeolite. It consists of disklike cavities, whose widths range from about $7.1 \AA$ in the circular 12 -membered ring windows to about 13 $\AA$ in the broadest regions of the channels $\left(R_{\mathrm{ctw}}=1.83\right)$. The cavities are connected in the $x$ direction only. although the windows connecting the cages are similar in size to those in AFI-type zeolite, the difference between the narrow parts and the broad parts of the channels is relatively large, and the system can be considered slightly cagelike, where one unit cell contains two LTL "cages". Industrially, LTL-type zeolites are used in aromatization reactions. ${ }^{88}$

On the cagelike side of the channel zeolites, we find SAStype zeolite. This zeolite is a one-dimensional version of the cage-type zeolite LTA. The cages (of about $10 \AA$ in diameter) are similar to LTA-cages, but they are connected in one dimension only, by eight-membered ring windows of about 4.2 $\AA$ in diameter, making $R_{\mathrm{ctw}}=2.38$. One unit cell of SAS-type zeolite contains two SAS cages. As the SAS topology has only recently been discovered, we are not aware of any industrial processes where zeolites of this type have found applications. ${ }^{65}$

Simulation of one-dimensional channels requires special attention. Here, diffusion results are very much dependent on the length of the channel, and surprisingly long channels are needed to reliably extrapolate to macroscopic diffusion coefficients. ${ }^{18}$

C. Intersecting Channel-Type Zeolites. The class of intersecting channel-type zeolites is also very diverse. These structures not only exist with a wide range of channel widths and window-to-cage ratios but also differ largely in the way the channels intersect. The channels not only intersect at various angles, frequencies, and channel numbers (either two or three channels can intersect at one point) but also intersect in different "amounts". The intersection can be "complete" (i.e., two (or 

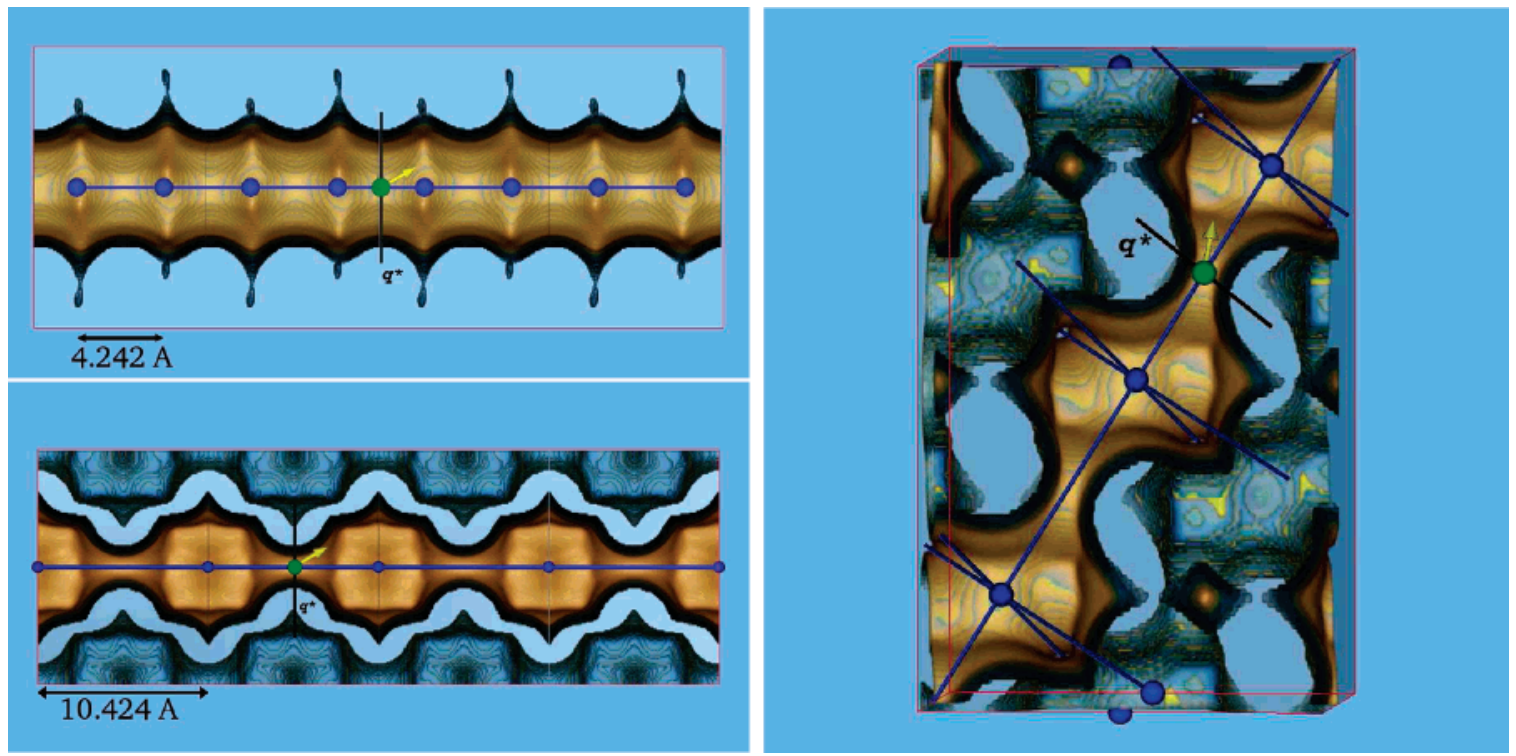

Figure 5. Lattice spanned by the wide parts of the tubes in AFI- and SAS-type zeolites and the cage centers of CHA-type zeolite. $q^{*}$ is the position of the barrier, perpendicular to the reaction coordinate. In the computation of $\kappa$, the particle starts in this plane.

three) channel axes intersect), or it can be "partial", meaning there is a hole large enough for a molecule to pass from one axis to the next, without the two channel axes intersecting exactly.

In this study, we look at four intersecting channel structures: MFI-, BOG-, BEC-, and ISV-type zeolites, of which the unit cell structures are depicted in Figure 4. The unit cell data are summarized in Table 1. For the intersecting channel-type zeolites it is not practical to talk about "cage" widths and window-tocage ratios. Usually the widest parts of the channels are regions where they are intersected by a perpendicular channel.

MFI-type zeolite is perhaps the most famous zeolite of this class. It is widely used industrially (as ZSM-5) because of its ability to promote hydrocarbon reactions. It consists of straight 10-ring channels, running in the $y$ direction, intersected (in complete intersections) by so-called zigzag channels that run in the $x$ and $z$ directions which also consist of 10-membered ring windows.

Boggsite (BOG-topology) is a naturally occurring mineral. Its channels run in the $x$ and $y$ directions only and intersect in partial intersections. The $x$-direction channels consist of 12 membered rings, while those running in $y$ direction contain 10membered rings.

BEC-type zeolite is one of the zeolite BETA polymorphs. It has 12-membered ring channels in the $x, y$, and $z$ directions. The channels in the $x$ and $y$ directions are equivalent, although the $z$ channels only intersect the channels running in the $x$ direction, in very broad complete intersections.

As a BEC-type zeolite, ISV consists of straight 12-membered ring channels in the $x, y$, and $z$ directions. Again, the channels in the $x$ and $y$ directions are equivalent, and the $z$ channels only intersect the channels running in the $x$ direction in broad complete intersections. However, in ISV-type zeolites, the channels running in the $z$ direction are merely short cross-links: they only connect two $x$ direction channels without continuing after the intersection.

D. Lattice Models in Zeolites. To be able to study diffusion using dcTST, it is necessary to choose a lattice to map on the zeolite structure. As shown in Figure 5, this is often straightforward, as it follows from the zeolite topology or from the free-energy profiles calculated from it. The lattices shown for AFI- and SAS-type zeolites are one-dimensional simple lattices, the lattice shown for CHA-type zeolite is a near-cubic lattice. It can be thought of as a cubic lattice that is slightly sheared in three directions to form angles of $94^{\circ}$. For all the other zeolites studied, a lattice can be constructed in a similar fashion.

We stress that the dcTST method we used is not a coarsegraining method. Knowledge of adsorption-site locations is not necessary to perform the simulation. The profiles are automatically averaged over all possible configurations of the system by calculating the free-energy profiles during a MD or MC run. Making use of the periodicity of nanoporous materials, we use a lattice point only as the integration region in the dcTST method to obtain a diffusion coefficient. ${ }^{32}$

\section{Results}

We calculated loading-dependent diffusion in cage-type zeolites (LTA, ERI, CHA, and FAU), channel-type zeolites (AFI, MTW, LTL, and SAS) and intersecting channel type zeolites (MFI, BOG, BEC and ISV). Free-energy profiles were calculated at different loadings, to help us understand the loading dependence of the diffusion. Free-energy differences play a role in both self and corrected diffusion. In this section, we discuss the self and corrected diffusion of methane in the three classes of zeolites. We show that while $D_{\mathrm{s}}$ can be computed directly from the free energy profiles in combination with the recrossing $\kappa$, all the details in the behavior of $D_{\mathrm{c}}$ as a function of loading can be explained very well by looking at these two parameters.

A. Self Diffusion. Self diffusion is a particle property. The self diffusion as a function of loading reflects the way in which the particle's diffusion is hampered by collisions or enhanced by the presence of other particles in favorable adsorption sites. We show the diffusion behavior in the three different zeolite classes.

1. Cage-Type Zeolites. We take LTA-type zeolite as a representative example of diffusion in cage-type zeolites, and we treat the diffusion of methane in LTA-type zeolite in detail. Although diffusion in this type of zeolite has been studied extensively, the diffusion behavior as a function of loading remains poorly understood. ${ }^{2,19,21-23,25}$ It has been shown to cause a maximum in the diffusion as a function of loading for a number of different molecules. 


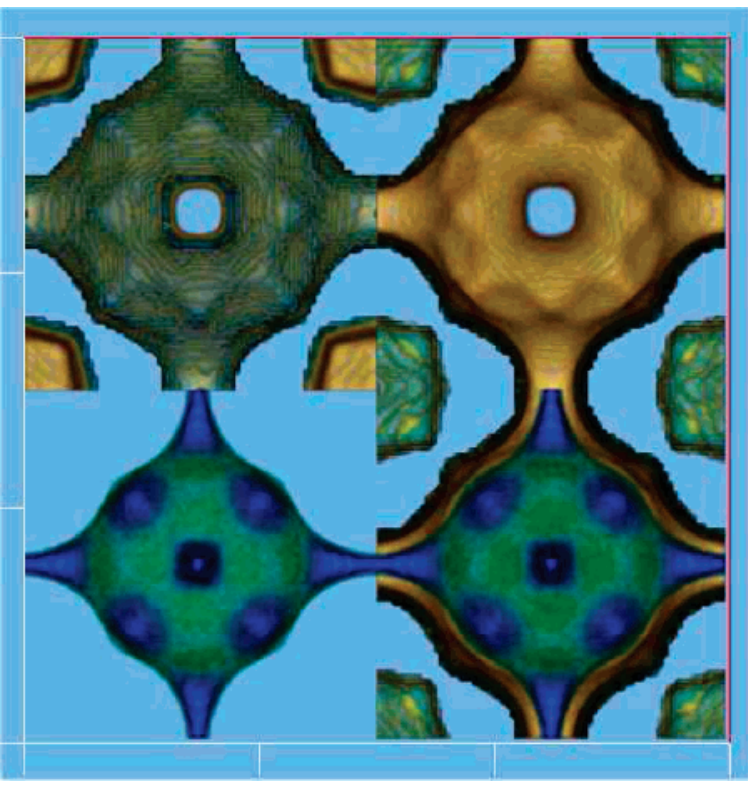

Figure 6. Density plot of the distribution of eight methane molecules per cage in LTA-type zeolite at $600 \mathrm{~K}$. Blue regions are favorable adsorption sites, green regions have a lower probability of containing a particle.

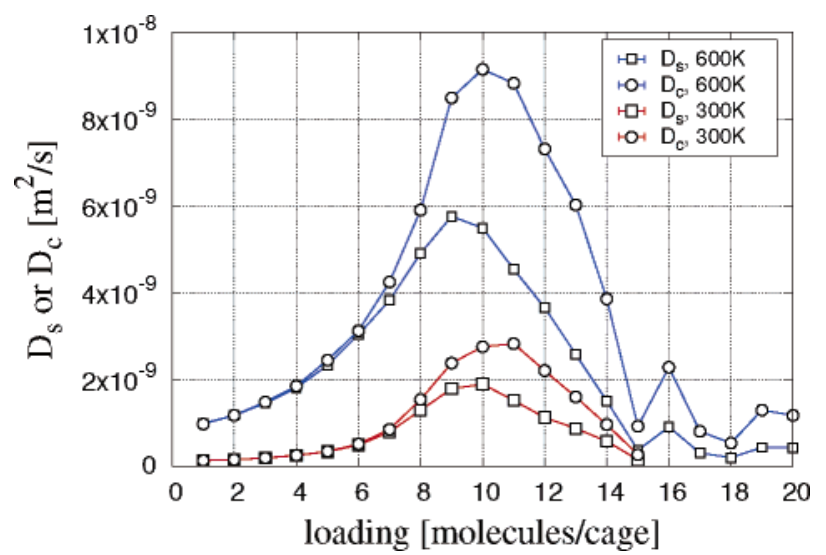

Figure 7. Self and collective diffusion of methane in a cage-type (LTA) zeolite at 300 and $600 \mathrm{~K}$.

A density plot of methane in LTA is shown in Figure 6. For small molecules, the positions in the window regions constitute six favorable adsorption sites. Other preferred positions are eight positions inside the cage, near the cage "wall", in regions with high curvature. Four of these are clearly visible in Figure 6, the other four are in a parallel plane straight behind, and obscured by, these four positions. These observations are in agreement with Demontis and Suffriti. ${ }^{89}$ One more preferred methane position is found exactly in the middle of the cage, and this makes the total number of preferred adsorption sites fifteen per cage.

Figure 7 shows the self and corrected diffusion of methane in LTA, as a function of loading. Both exhibit a maximum at a loading of about 9-11 molecules per cage and a minimum at 15 molecules per cage, the loading at which all 15 preferred adsorption sites are occupied. There is a clear difference between $D_{\mathrm{s}}$ and $D_{\mathrm{c}}$, especially at intermediate loadings, $D_{\mathrm{c}}>D_{\mathrm{s}}$, caused by interparticle or back correlations increasing $D_{\mathrm{c}}$ and diminishing $D_{\mathrm{s}}$.

To explain this, we look at the free-energy profiles of methane in LTA, calculated at various loadings (see Figure 8 (top)). Plotted is the free energy as a function of loading across two LTA cages. The lower parts of these profiles correspond to the
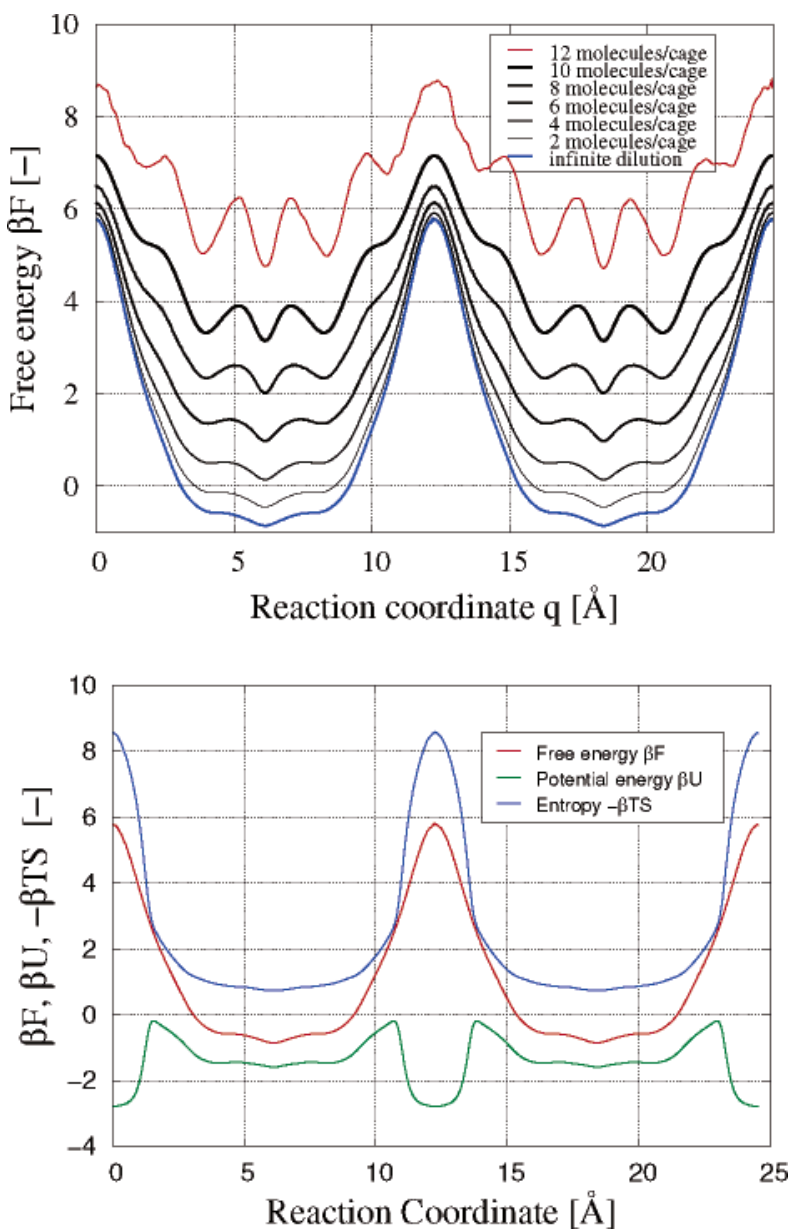

Figure 8. (top) Free-energy profiles as a function of loading at $600 \mathrm{~K}$ for methane in a cage-type (LTA) zeolite. (bottom) Free-energy profile, $\beta F=\beta U-\beta T S$, for methane in the empty zeolite with potential energy contribution, $\beta U$, and entropy contribution, $\beta T S=S / k_{\mathrm{B}}$, as plotted by Shüring et al..$^{90}$

interior of the cages; the maximum in the free-energy graphs (around $12.2 \AA$ ) is located at the windows connecting two LTA cages. Because a high value of the free energy corresponds to a low probability of occupying this position, one might be tempted to conclude from the profiles in Figure 8 (top) that the window between two cages is an unfavorable adsorption region. However, because the free energy, calculated in a slice perpendicular to the reaction coordinate (here the $x$ coordinate), is a function of both the potential energy and the entropy, this is not necessarily true. In our example, the slice perpendicular to the window consists of a narrow low-energy window region and a large amount of inaccessible "zeolite wall". Figure 8 (bottom) shows the free-energy profile for methane in LTA, at zero loading, together with the potential-energy profile and the entropy $(T S)$ profile. The free energy of the empty zeolite was calculated by performing Widom particle insertion and computing the Boltzmann factors for the separate interactions, in the following way

$$
\beta F(q)=-\ln \left(\left\langle\mathrm{e}^{(-\beta U)}\right\rangle_{q}\right)
$$

where $F(q)$ is the free energy of the particle-zeolite interaction at position $q$ and $U$ is the particle-zeolite interaction energy.

The potential energy as a function of the reaction coordinate was calculated using 


$$
U(q)=\langle U\rangle_{q}=\frac{\sum_{x, y}\left(U(x, y, q) \mathrm{e}^{(-\beta U(x, y, q))}\right)}{\sum_{x, y} \mathrm{e}^{(-\beta U(x, y, q))}}
$$

(i.e., $\langle U\rangle_{q}$ is the average energy at a certain value of the reaction coordinate $q$, calculated in a plane perpendicular to the reaction coordinate). The entropy, expressed as $T S(q)$, was obtained from $F(q)=U(q)-T S(q)$.

When the minimum in the potential energy is considered, it is clear that the window between the two cages is a favorable adsorption position. However, since this region is so narrow, the window is an entropic barrier, as expressed by a high value of $-T S$ in the barrier region.

As the loading increases, more and more particles enter the cages. The surface of the LTA cage is adsorbophilic: adding a molecule causes a decrease in the interaction with the walls. This favorable interaction is being replaced by a less favorable interaction with other particles, and hence, in Figure 8 (top) we observe an increase of the free energy in the bottom of the well. Meanwhile, the free energy in the window region barely changes, so that while the zeolite is being filled up, the net freeenergy barrier decreases, causing an increase in the diffusion coefficient.

At about 10 molecules per unit cell, the free-energy barrier starts to decrease again because of packing effects. As the zeolite fills up, the molecules inside the cages become increasingly ordered. New free-energy barriers arise, and the diffusion slows down. At a loading of 15 molecules per unit cell, the methane molecules follow a highly ordered pattern inside the cages, every preferable position that is indicated in Figure 6 is now occupied. The addition of another molecule means that this ordered structure has to be disturbed and a new ordering has to be formed. This new ordering changes the shape of the free-energy profile, and the diffusion increases again. Again, the molecule positions in the new ordering fill up, the diffusion increases a bit and afterward decreases again, causing a second, smaller, maximum in Figure 7.

We note that the new ordering at high loading and the second maximum in the diffusion graph will be very difficult to observe experimentally, because it would require very high pressures.

As explained in the Methods section, transition state theory can be used to calculate a hopping rate, $k^{\mathrm{TST}}$, for the diffusion from cage to cage from the free-energy profiles. The true hopping rate, $k$, is then obtained by multiplying this $k^{\mathrm{TST}}$ value with a factor $\kappa$ that contains corrections for a nonideal choice of reaction coordinate and collisions and correlations with other particles. Since LTA-type cages are highly symmetric, it is possible to define a perfect reaction coordinate (i.e., at zeroloading, $\kappa$ equals 1 ). At higher loadings, the deviations in $\kappa$ are caused solely by interparticle collisions and correlations. It is therefore possible to split the diffusion of methane in LTAtype zeolite in a free-energy part and a collision part. The freeenergy part is given by the transition state hopping rate from cage to cage, which is calculated directly from the free-energy profiles. The collision part can be obtained by calculation of $\kappa$. Figure 9 shows the two contributions as a function of loading. As the loading increases the transition state hopping rate at first increases to reach a maximum at around 11 molecules per unit cell. It is at this loading that we observe a sharper increase of the free energy at the barrier and the creation of new free-energy barriers inside the cage, caused by an increased ordering of the particles. While the free-energy part of the diffusion thus shows

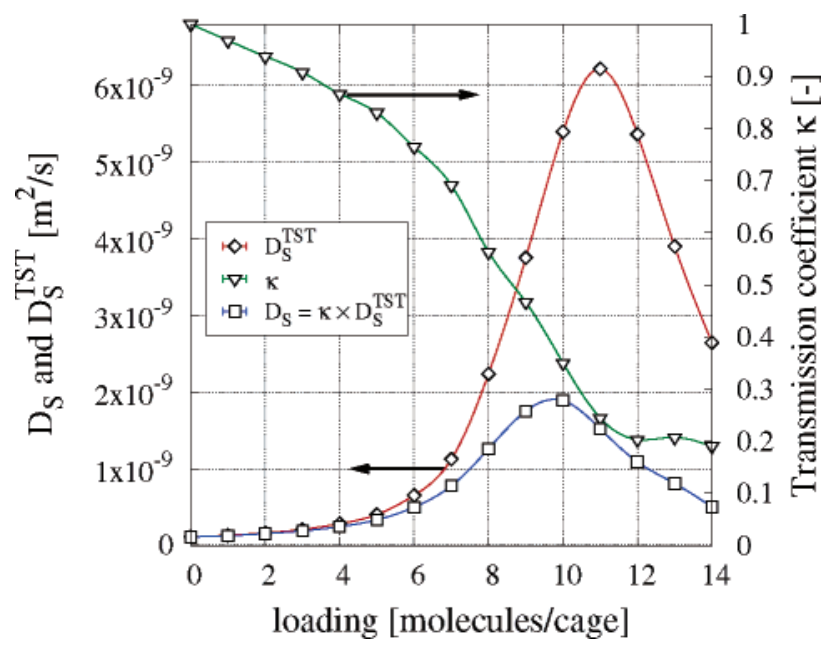

Figure 9. Diffusion of methane in LTA-type zeolite as a function of loading at $300 \mathrm{~K}$ (left axis) and the two contributions to this diffusion: the free-energy part, given by the transition state self-diffusion coefficient, $D^{\text {TST }}$ (left axis), and the collision part, given by the transmission coefficient, $\kappa$ (right axis).

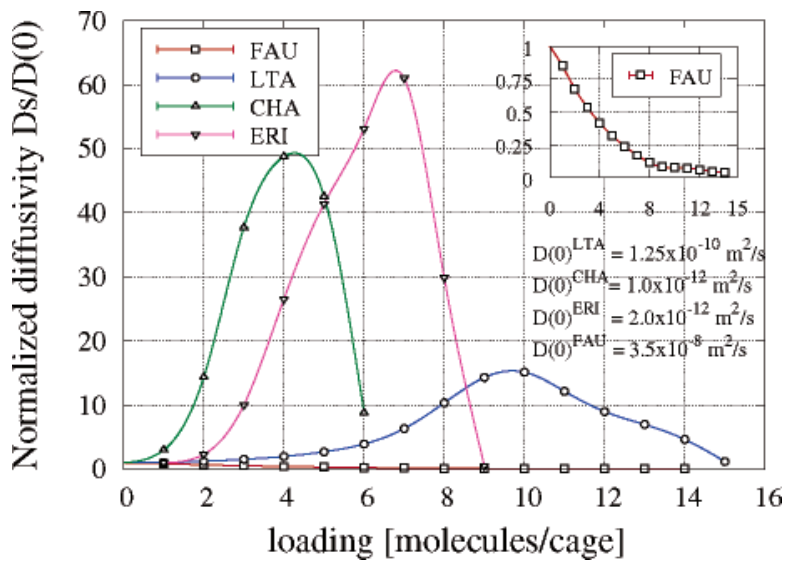

Figure 10. Normalized self diffusion of methane in cage-type zeolites ERI, LTA, CHA, and FAU, as a function of loading at $300 \mathrm{~K}$. The inset shows the normalized self diffusion in FAU.

a maximum as a function of loading, the collision term, represented by $\kappa$ is a continuously decreasing function of the loading because, as the number of particles in the system increases, collisions become ever more frequent events. Figure 9 clearly shows that the self diffusion coefficient follows the trend of $k^{\mathrm{TST}}$. In other words, the qualitative diffusion behavior is determined by the change in free energy profiles. The collision factor $\kappa$ has a quantitative effect on the diffusion coefficient and shifts the maximum in the diffusion curve slightly toward lower loadings.

Simulations of methane diffusion in different cage/windowtype zeolites show that the observed diffusion behavior is not specific for LTA-type zeolites but is typical of this class of zeolite structures. Figure 10 shows diffusion as a function of loading in LTA, ERI, CHA, and FAU. The loading at which the maximum in the diffusion coefficient is observed is dependent on the cage size. Clearly, if we increase the size of the cage, the position of the maximum in the diffusion coefficient will change accordingly. This is exactly what we observe for CHA, ERI, and LTA: CHA has the smallest cages of the three, and LTA has the largest. We note that this fact is also reflected in the value for the diffusion at zero loading (given in Figure 10): of these three zeolite types, the highest diffusion in the infinite dilution limit is observed in LTA, followed by ERI, and subsequently CHA. The increase in self diffusion 


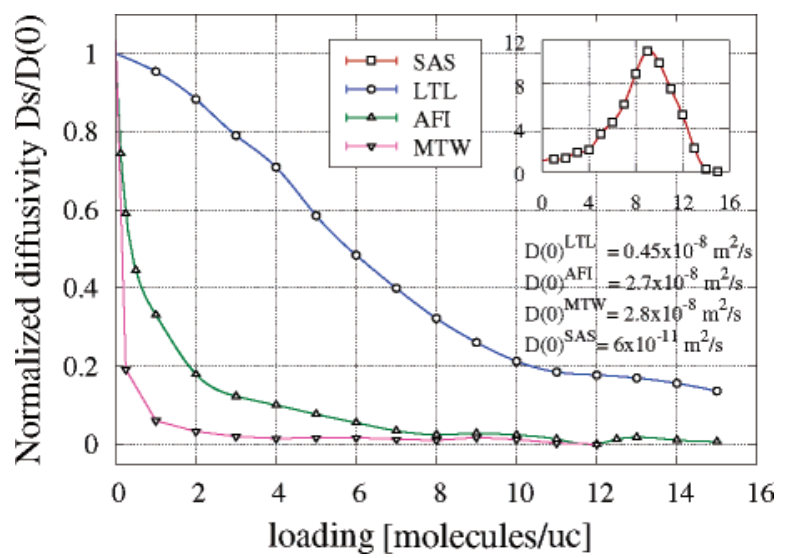

Figure 11. Normalized self diffusion of methane in channel-type zeolites AFI, MTW, LTL, and SAS (inset), as a function of loading at $300 \mathrm{~K}$.

compared to the infinite dilution limit can be very large, depending mainly on the size of the window and thus the height of the free-energy barrier. Especially in the case of ERI-type zeolite (whose $3.6 \AA$ windows are the narrowest of the three), we observe an increase in the self diffusion by a factor of 60 . CHA and LTA have slightly wider windows, of 3.8 and $4.1 \AA$, respectively, and accordingly, the maximum in the normalized self diffusion is smaller than that in ERI-type zeolite.

The diffusion in FAU type (Figure 10 (inset)) is quite different from that in the other three cage-type zeolites. While the initial slope is positive in the case of ERI, CHA, and LTA, the self diffusion of methane in FAU-type zeolites is a continuously decreasing function of loading. The reason for this is the large size of the windows that connect the FAU cages. The diameter of these windows, about $7.1 \AA$, does not differ much from the diameter of the cages, about $9 \AA$, and thus the windows hardly form a barrier for the diffusion. Since both the cages and the windows are large, the FAU zeolite structure forms a very weak confinement, the free-energy profiles barely change when the loading is increased, and the diffusion as a function of loading is as expected in a system of particles hopping on a lattice where the lattice does not change with loading. Since the diffusion in FAU-type zeolite is so much different from that in other cagetype zeolites, we will treat it in more detail in section V.C.

2. Channel-Type Zeolites. The diffusion behavior of methane in these systems is highly dependent on the ratio of the widest parts of the channels to the narrowest parts of the channels, the window-to-cage diameter ratio or $R_{w t c}$.

The qualitative behavior is very different compared to that in cage-type zeolites. Whereas in the LTA-type zeolite, we observe a maximum in the diffusion as a function of loading, in the smooth channels of this class of zeolites, the diffusion slows down when the loading is increased.

An explanation of this behavior can be found in the freeenergy profiles of methane in the AFI-type zeolite (Figure 12). The minimums and maximums in the profiles correspond to the broader and narrower parts of the AFI tubes, respectively. At zero loading, the height of the effective free-energy barriers is about $1.5 k_{\mathrm{B}} T$. Such a low value implies that the diffusion in $z$ direction is barely hampered by the zeolite structure. Unlike in the cage-type zeolites, up to a loading of about 12 molecules per unit cell, the free-energy barrier increases, rather than decreases, when the loading is increased. Apparently, at higher loading, a larger portion of the particles is located in the wider regions of the AFI channels. At a loading of about 12 molecules per unit cell, however, the form of the free-energy profiles
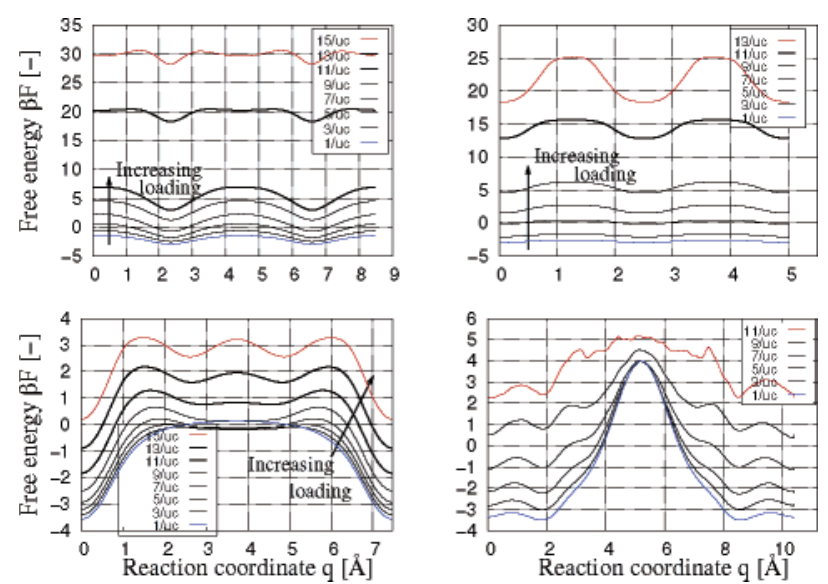

Figure 12. Free-energy profiles at $300 \mathrm{~K}$ at different loadings of methane for AFI- (top left), MTW- (top right), LTL- (bottom left), and SAS-type zeolites (bottom right).

changes. This is in agreement with Maris et al. ${ }^{91}$ who found a reordering of methane molecules in AFI-type zeolites at higher loadings. In the altered form of the free-energy profile, the freeenergy barrier is much lower, and this produces an acceleration of the diffusion, as is indeed observed at loadings higher than 12 molecules per unit cell.

When we compare the diffusion of methane in AFI-type zeolite with that in a one-dimensional channel-type structure with a similar $R_{\text {wtc }}$ (e.g., MTW-type zeolite), we observe similar diffusion behavior (see Figure 11). Common to these two systems is their preference for a fixed ordering at lower loadings (while this particle arrangement fills up when the loading is increased, the diffusion decreases) and a sudden change of particle positions at higher loadings, which gives rise to a peak in the diffusion as a function of loading. Because $R_{\mathrm{ctw}}$ in MTWtype zeolite is a bit higher than that in AFI-type zeolite, the free-energy barriers in MTW-type zeolite (shown in Figure 12) are higher and the low-loading behavior of the diffusion is slightly more cagelike in nature, which is expressed in a slightly convex initial slope of the corrected diffusion as a function of loading, as will be discussed in section V.B. However, since the distance between two consecutive free-energy barriers in MTW-type zeolite is much smaller than in AFI-type (5.012 vs $8.484 \AA$ ), the back-correlations are much higher, as evidenced by a very low self diffusion.

In LTL-type zeolite, because the windows are quite broad, the dominant behavior of methane diffusion is that of methane in smooth linear tubes, such as AFI-type zeolite. However, since the difference in width between the windows and the cage regions is large (i.e., the cages are even broader), at lower loadings, we still observe some cagelike behavior: the self diffusion of methane is a slightly convex function of loading. The cagelike behavior becomes more evident in the corrected diffusion, in section V.B.

On the cagelike side of the channel zeolites, we find SAStype zeolite. When the topology, which consists of onedimensionally connected LTA-cages, is considered, it is not surprising that the diffusion of methane in the SAS-type zeolite follows a trend similar to that in three-dimensional cage-type zeolites such as LTA, CHA, and ERI: an increase in the diffusion up to a loading of about half the maximum loading, caused by an increase in the free energy inside the cages (see Figure 12 (bottom, right)), followed by a decrease that approaches zero at the maximum loading. Note that, although the cages of SAS-type zeolite are comparable to those in LTAtype zeolite, the maximum loading is a bit lower than in LTA- 


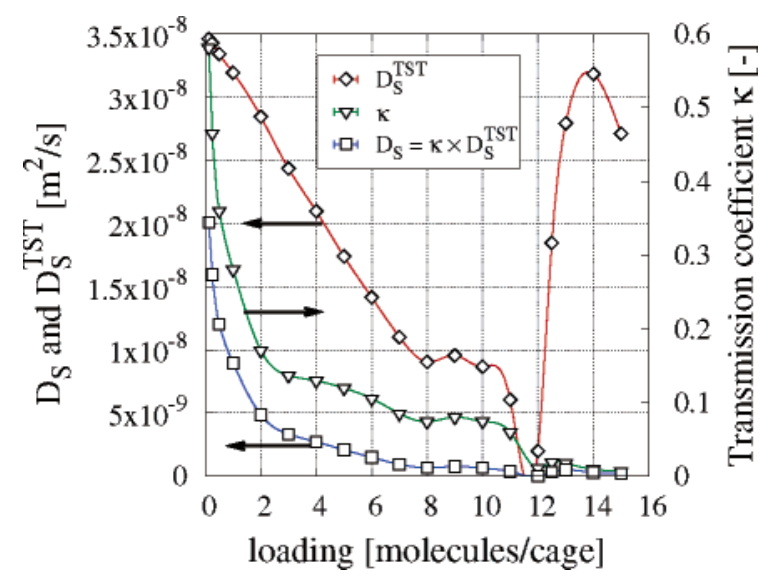

Figure 13. Diffusion of methane in AFI-type zeolite as a function of loading (left axis) at $300 \mathrm{~K}$ and the two contributions to this diffusion: the free-energy part, given by the transition state self-diffusion coefficient, $D^{\text {TST }}$ (left axis), and the collision part, given by the transmission coefficient, $\kappa$ (right axis).

type zeolite. The particles are arranged differently because of the lack of windows in four directions (out of the six available in LTA-type zeolite) and the fact that the connection between the cages is different than that in LTA-type zeolite.

In summary, the self diffusion in channel-type zeolites is dependent mainly on $R_{\mathrm{wtc}}$, the height of the free-energy barriers, and the distances between them. When we split the Diffusion coefficient in two contributions, $D_{\mathrm{s}}^{\mathrm{TST}}$ and $\kappa$, as shown for AFItype zeolite in Figure 13, we see that the diffusion is influenced by both contributions: $D_{\mathrm{s}}^{\mathrm{TST}}$ is a steadily decreasing function of loading, but $\kappa$ also decreases rapidly. As a consequence, the self diffusion of methane in AFI-type zeolite is a steeply decreasing function of loading, with a small maximum around 12 molecules per unit cell.

Figure 14 (top) shows the $D_{\mathrm{s}}^{\mathrm{TST}}$ component of the diffusion for AFI-, MTW-, LTL-, and SAS-type zeolites. For SAS-type zeolites, we clearly see the same cagelike behavior we saw earlier for LTA-type zeolite. At low loadings, the effective freeenergy barrier decreases, and the free-energy part of the diffusion increases. Also for LTL-type zeolite, $D_{\mathrm{s}}^{\mathrm{TST}}$ is an increasing function of loading, although now it is continuously increasing because of the broad barriers depicted in Figure 12 (bottom, left). Only for the two smooth channels, AFI and MTW, does the $D_{\mathrm{s}}^{\mathrm{TST}}$ value decrease as a function of the loading. Figure 14 (bottom) shows the $\kappa$ component of the diffusion. As expected, the value of $\kappa$ is highest in the high-barrier system of SAStype zeolite, where interparticle correlations are low, and the lowest in MTW, which has the smallest distance between two consecutive barriers and therefore the highest interparticle correlations. LTL- and AFI-type zeolites have a value of $\kappa$ between these two extremes. This leads to the conclusion that from a free-energy point of view SAS and LTL are cage-type zeolites, whereas AFI and MTW are not. From an interparticle correlations point of view, SAS is a cage-type zeolite, MTW clearly is not, and AFI and LTL are something in between. This results in cage-type diffusion behavior in SAS-type zeolites, slightly cage-type behavior in LTL, and smooth-channel behavior in AFI and MTW.

3. Intersecting Channel-Type Zeolites. An important class of zeolites, is the class of intersecting channel-type zeolites, of which MFI is the most well-known example. These structures consist of three-dimensionally interconnected straight channels. The self diffusion of methane in four of these structures, MFI, BOG, BEC, and ISV, is shown in Figure 15. This figure shows
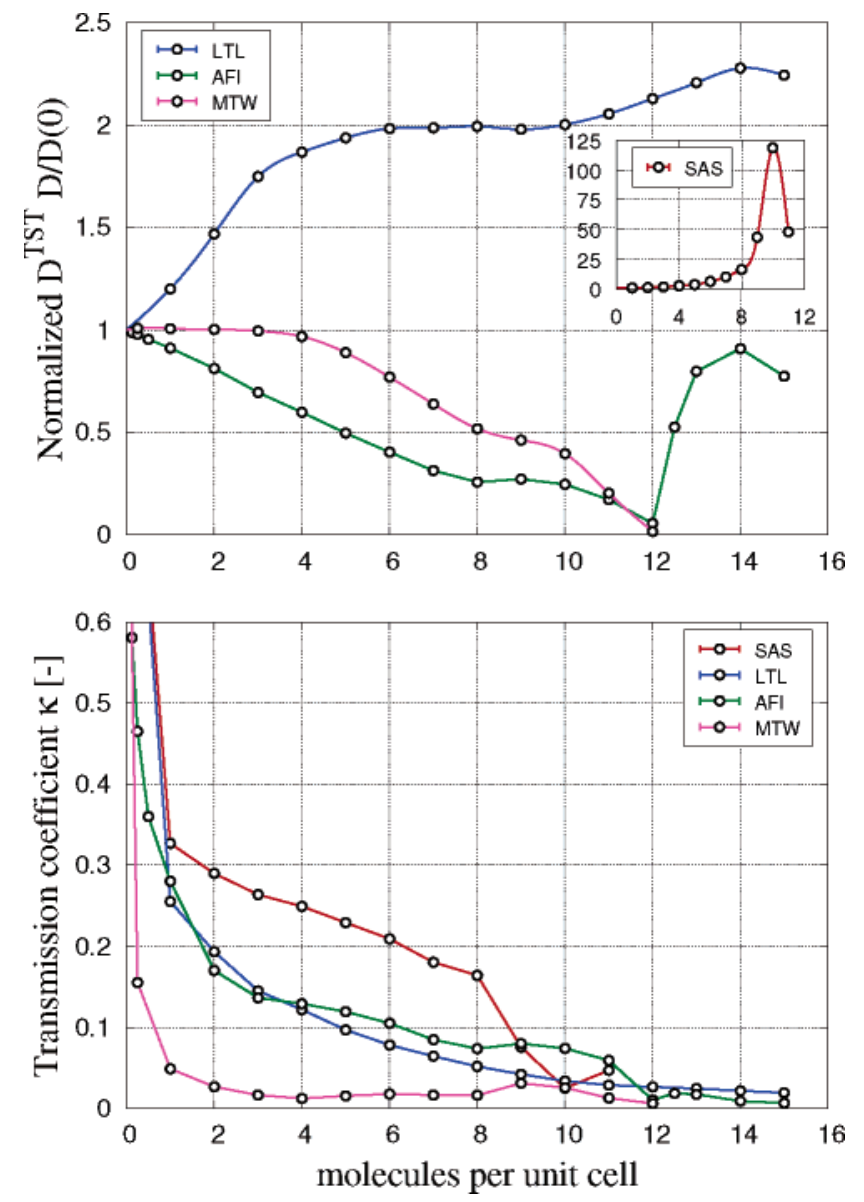

Figure 14. (top) Normalized free-energy contribution, $D^{\mathrm{TST}}$, for AFI-, MTW-, LTL-, and SAS-type (inset) zeolites and (bottom) collision contribution, $\kappa$, for the four structures.

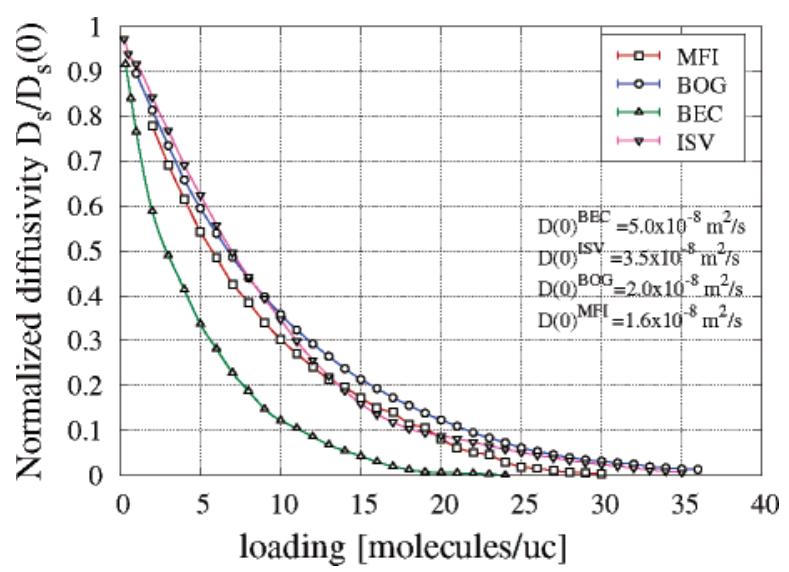

Figure 15. Normalized self diffusion of methane in intersecting channel-type zeolites MFI, BOG, BEC, and ISV as a function of loading at $300 \mathrm{~K}$.

the diffusion relative to that in the infinite dilution limit. It is a monotonically decreasing function of loading, similar to that in smooth channel-type zeolites such as AFI and MTW. The self-diffusion behavior of methane in MFI-, ISV-, and BOGtype zeolites is almost identical because the maximum loadings of the three zeolites are similar. The diffusion in BEC-type zeolite is also very similar to the other intersecting channeltype zeolites, although the maximum loading is about half that of MFI, BOG, and ISV, in agreement with the relative size of the unit cell. The unit cell is smaller, and therefore the diffusion behavior is shifted toward lower loadings.

The value of the diffusion coefficients in the infinite dilution 

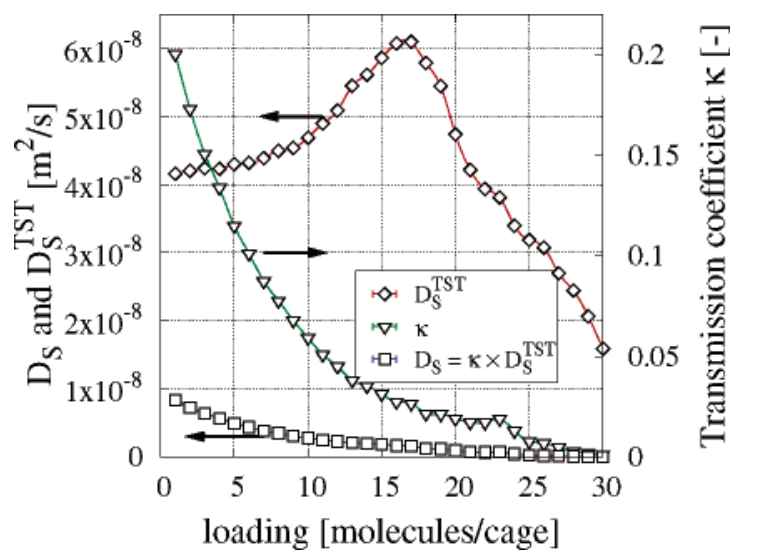

Figure 16. $D_{\mathrm{s}, y}$ of methane in the MFI-type zeolite at $300 \mathrm{~K}$ as a function of loading (left axis) and the two contributions to this diffusion in the $y$ direction: the free energy part, given by the transition state self-diffusion coefficient, $D_{y}^{\mathrm{TST}}$ (left axis), and the collision part, given by the transmission coefficient, $\kappa_{y}$ (right axis).

limit is dependent mainly on the smallest ring-size of the zeolite channels. $D_{0}^{\mathrm{BEC}}>D_{0}^{\mathrm{ISV}}>D_{0}^{\mathrm{BOG}}>D_{0}^{\mathrm{MFI}}$, while BEC- and ISVtype zeolites have a smallest ring size of 12 in both the straight channels and the perpendicular channels; BOG-type zeolites have 12-membered rings in the $x$ direction and 10-membered rings in the narrower $y$ direction channels. MFI-type zeolites have 10-membered rings in both the straight and the zigzag channels. However, the exact value of $D_{0}$ depends on the exact topology and cannot be determined a priori by the ring size alone.

As an example, we split $D_{\mathrm{s}}^{\mathrm{TST}}$ into $D^{\mathrm{TST}}$ and $\kappa$ for diffusion in MFI-type zeolite along the $y$ direction (see Figure 16). From the behavior of $D_{\mathrm{s}}^{\mathrm{TST}}$, one could expect a cagelike behavior of the diffusion. However, it is clear that the diffusion is completely dominated by $\kappa$, which is a measure of the correlations in the system and the collision frequency. $\kappa$ decreases more rapidly than $D^{\text {TST }}$ decreases, so the net effect is that the self diffusion in MFI-type zeolite is a decreasing function of loading. The cage-type suggested by the maximum in $D_{\mathrm{s}}^{\mathrm{TST}}$ is more visible in the loading behavior of the corrected diffusion. We note that the figure shows that $\kappa$ is not equal to 1 at zero loading, which implies that $\kappa$ also contains some corrections for a nonideal choice of reaction coordinate.

B. Corrected Diffusivity. The corrected diffusion behavior is a system property. It is the collective diffusion of all particles through the zeolite and includes interparticle correlations. As it turns out, corrected diffusion as a function of loading can be described accurately by looking at the free-energy profiles as a function of loading. Again, each of the three zeolite classes has a very distinct loading-dependent diffusion behavior.

1. Cage-Type Zeolites. Figure 17 shows the corrected diffusion of methane in LTA, CHA, ERI, and FAU, normalized with respect to the diffusion in the infinite dilution limit. For LTA-, CHA-, and ERI-type zeolites, the qualitative diffusion behavior is similar to the self diffusion: a maximum as a function of loading at about $2 / 3$ of the maximum loading. As expected, the corrected diffusion is higher than the self diffusion because of interparticle and back correlations. Since at zero loading the self diffusion is equal to the corrected diffusion, $D_{0}^{\mathrm{FAU}}>D_{0}^{\mathrm{LTA}}>D_{0}^{\mathrm{ERI}}>D_{0}^{\mathrm{CHA}}$ also holds for the corrected diffusion.

The increase in the corrected diffusion compared to the infinite dilution limit is even higher than that in the self diffusion. In ERI- and CHA-type zeolites, the increase in the diffusion is almost 2 orders of magnitude.

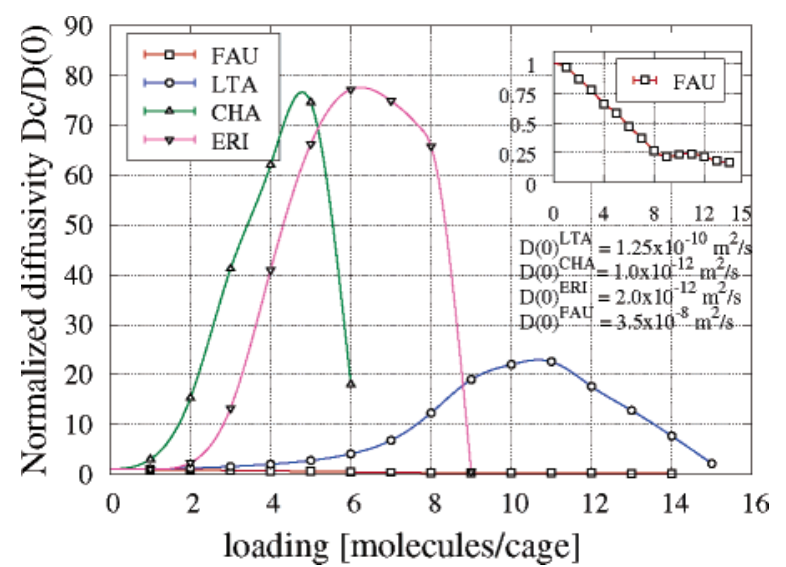

Figure 17. Normalized corrected diffusion of methane in cage-type zeolites LTA, CHA, ERI, and FAU as a function of loading at $300 \mathrm{~K}$.

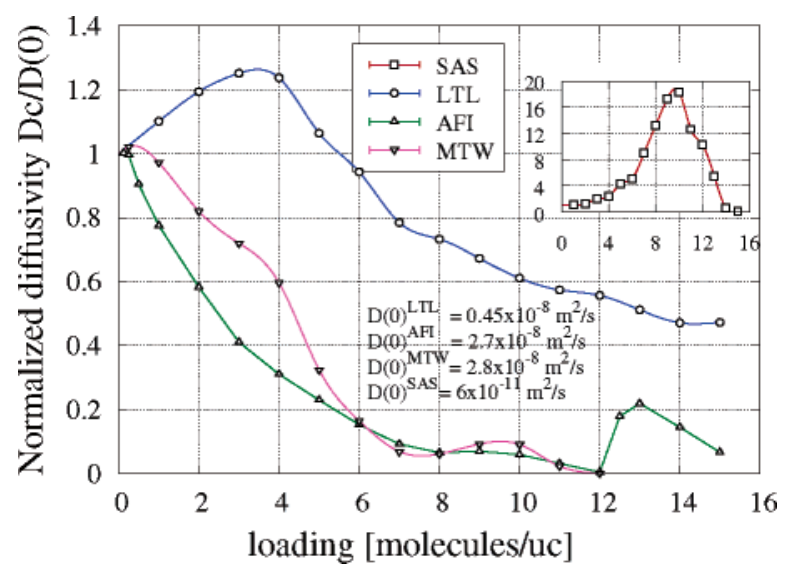

Figure 18. Normalized corrected diffusion of methane in channeltype zeolites AFI, MTW, LTL, and SAS (inset) as a function of loading at $300 \mathrm{~K}$.

Again, the diffusion in FAU-type zeolite is completely different from the diffusion in the other cage-type zeolites. It is an almost linearly decreasing function of loading, as expected of corrected diffusion in a system where the barriers do not change as a function of the loading. The corrected diffusion of the FAU-type zeolite will be treated in more detail in section V.C.

2. Channel-Type Zeolites. As in the case of self diffusion, the diffusion behavior as a function of loading is highly dependent on the amount of cagelikeness, for which $R_{\mathrm{wtc}}$ is a good indicator. For our four-channel-type zeolites, $R_{\mathrm{wtc}}^{\mathrm{SAS}}>$ $R_{\mathrm{wtc}}^{\mathrm{LTL}}>R_{\mathrm{wtc}}^{\mathrm{MTW}}>R_{\mathrm{wtc}}^{\mathrm{AFI}}$, and this is clearly reflected in the diffusion curves in Figure 18. The diffusion of methane in AFIand MTW-type zeolites is a continuously decreasing function of loading. The curve is more convex for MTW because of the higher $R_{\mathrm{wtc}}$. As in the case of self diffusion, a small maximum is found at about 10 and 13 molecules per unit cell, in MTW and AFI, respectively, because of a reordering of adsorbed molecules inside the zeolite.

In LTL, the difference in width between the windows and the cage regions is quite large, and therefore, at low loadings, we still observe some cagelike behavior in the corrected diffusion. There is a small peak in the diffusion at a loading of about 3 molecules per unit cell. When the loading is further increased, the diffusion slows down, as is expected in a tubelike structure. As in the case of self diffusion, SAS-type zeolite clearly exhibits cage-type behavior.

The corrected diffusion of methane in channel-type zeolites SAS, LTL, MTW, and AFI is shown in Figure 18. 


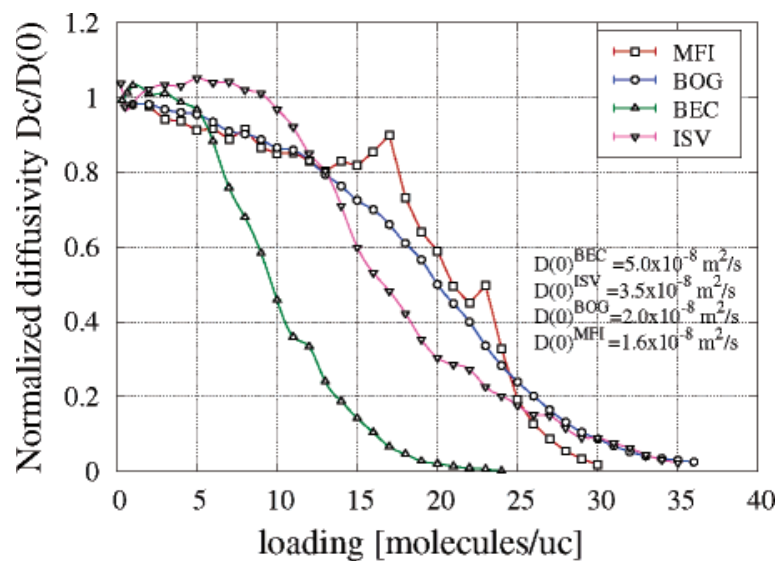

Figure 19. Normalized corrected diffusion of methane in intersecting channel-type zeolites MFI, BOG, BEC, and ISV as a function of loading at $300 \mathrm{~K}$.

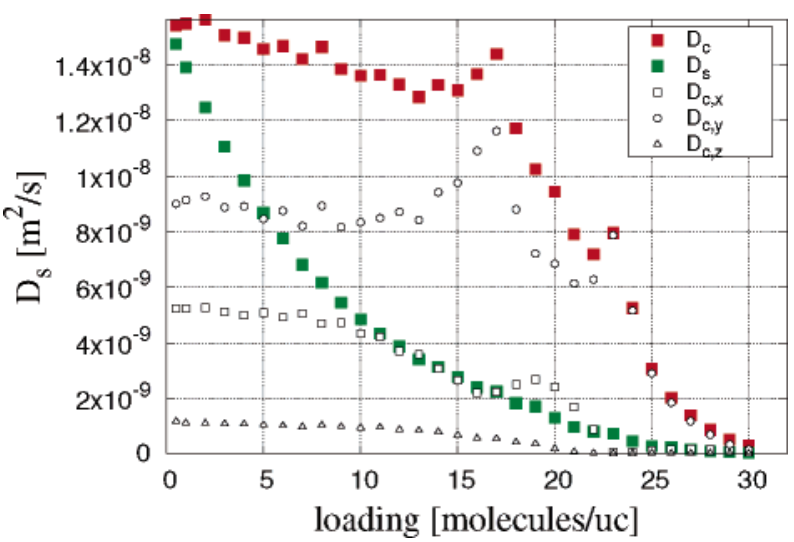

Figure 20. $D_{\mathrm{s}}$ and $D_{\mathrm{c}}$ of methane in MFI-type zeolite, as a function of loading, $D_{\mathrm{c}}$ in the $x, y$, and $z$ directions.

3. Intersecting Channel-Type Zeolites. As an example of how the free-energy profiles can contain a lot of detail about diffusion behavior, we treat the diffusion in MFI-type zeolite in detail. The structure of MFI consists of two straight channels per unit cell, intersected by two zigzag channels. At low loadings, there are four favorable positions per straight channel and four favorable positions per zigzag channel, making the total number of preferential adsorption sites per unit cell 16 .

Pascual et al. found that at low loadings, methane in the MFItype zeolite does not have any preference for either of these positions and is located everywhere inside the zeolite. ${ }^{92}$ When the loading exceeds 16 molecules per unit cell, all these positions are filled, and new adsorption sites have to be formed to accommodate more molecules.

Again, our explanation of the diffusion behavior comes from free-energy profiles, shown in Figure 21. The $x, y$, and $z$ directions in MFI-type zeolite are inequivalent; therefore we have to consider them separately.

The fastest diffusion occurs along the $y$ direction in the straight channels. At low loadings (up to about 13 molecules per unit cell), the particles are so far apart that they hardly influence each other's diffusion. $D_{\mathrm{c}, y}$ is approximately constant, and the free-energy profiles remain the same. The highest freeenergy barrier is located at $\mathrm{B}$. The minima in the free-energy profiles (at about 5 and $15 \AA$ ) correspond to the positions of the intersections and zigzag channels (all mapped onto the same $y$-coordinate); the region of 5-15 $\AA$ and the region of $15-20$ and $0-5 \AA$ correspond to the interior of the straight channels. Apart from the intersections, there are four minima in the free-
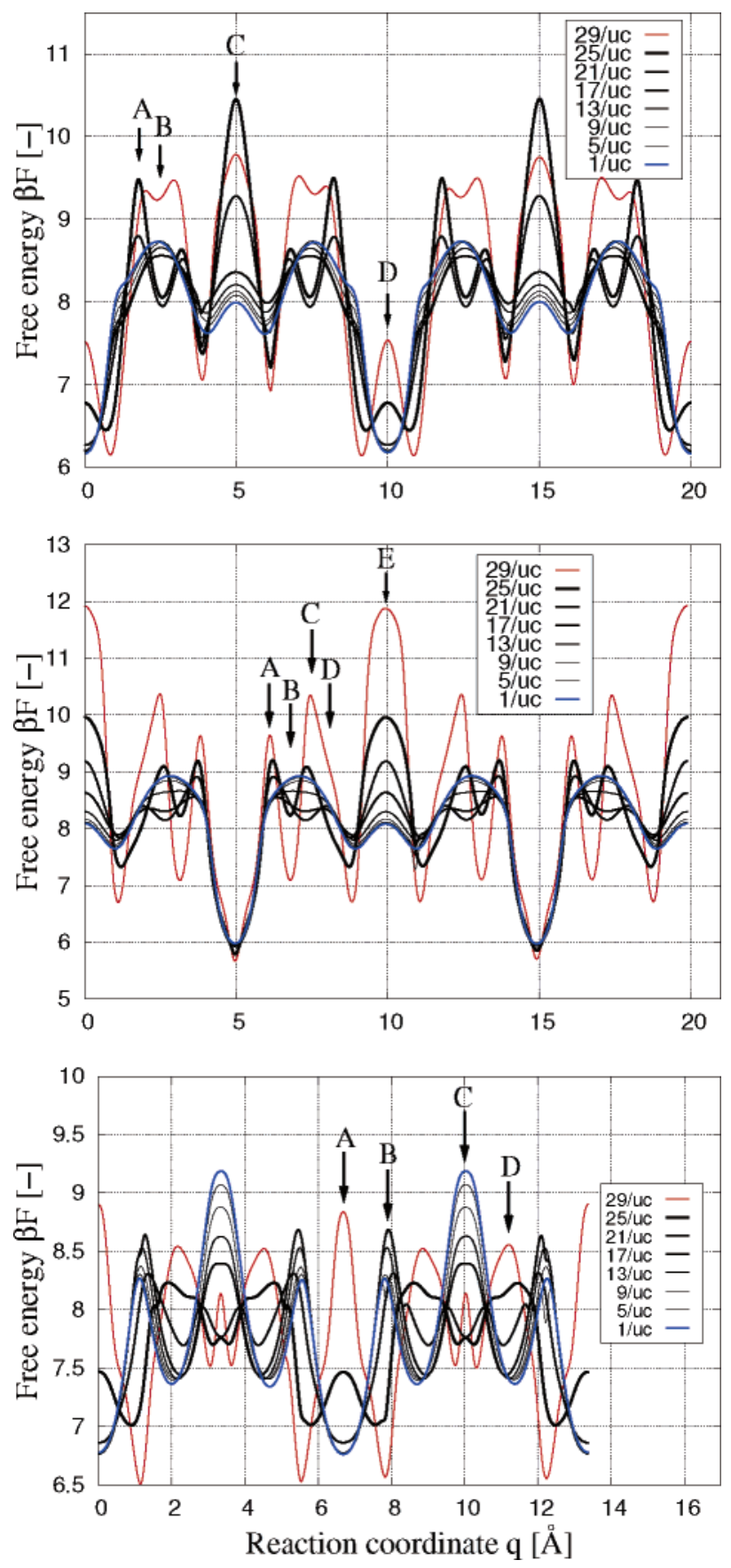

Figure 21. Free-energy profiles, $300 \mathrm{~K}$, at different loadings for methane in MFI-type zeolite along the $x, y$, and $z$ directions, respectively. The straight channels run in the $y$ direction and the zigzag channels run in the $x$ and $z$ directions.

energy profiles along the direction of the straight channels, which correspond with four favorable adsorption positions.

When the loading exceeds 13 molecules per unit cell, the free-energy profile changes slightly (see Figure 22 (top)): the barrier at B is lowered, and the smaller barrier at D is raised a bit. This indicates that, while the "most common" configuration is still four adsorption sites per straight channel, the average loading of the straight channels increases, and some molecules are located at nonstandard positions inside the straight channel. The observed free-energy profile is an average over all these configurations, and as the net barrier decreases, we see a slight increase in the diffusion in the $y$ direction, between 13 and 17 molecules per unit cell. At a loading of 17 molecules per unit cell and higher, the dominant change in the free-energy profiles 

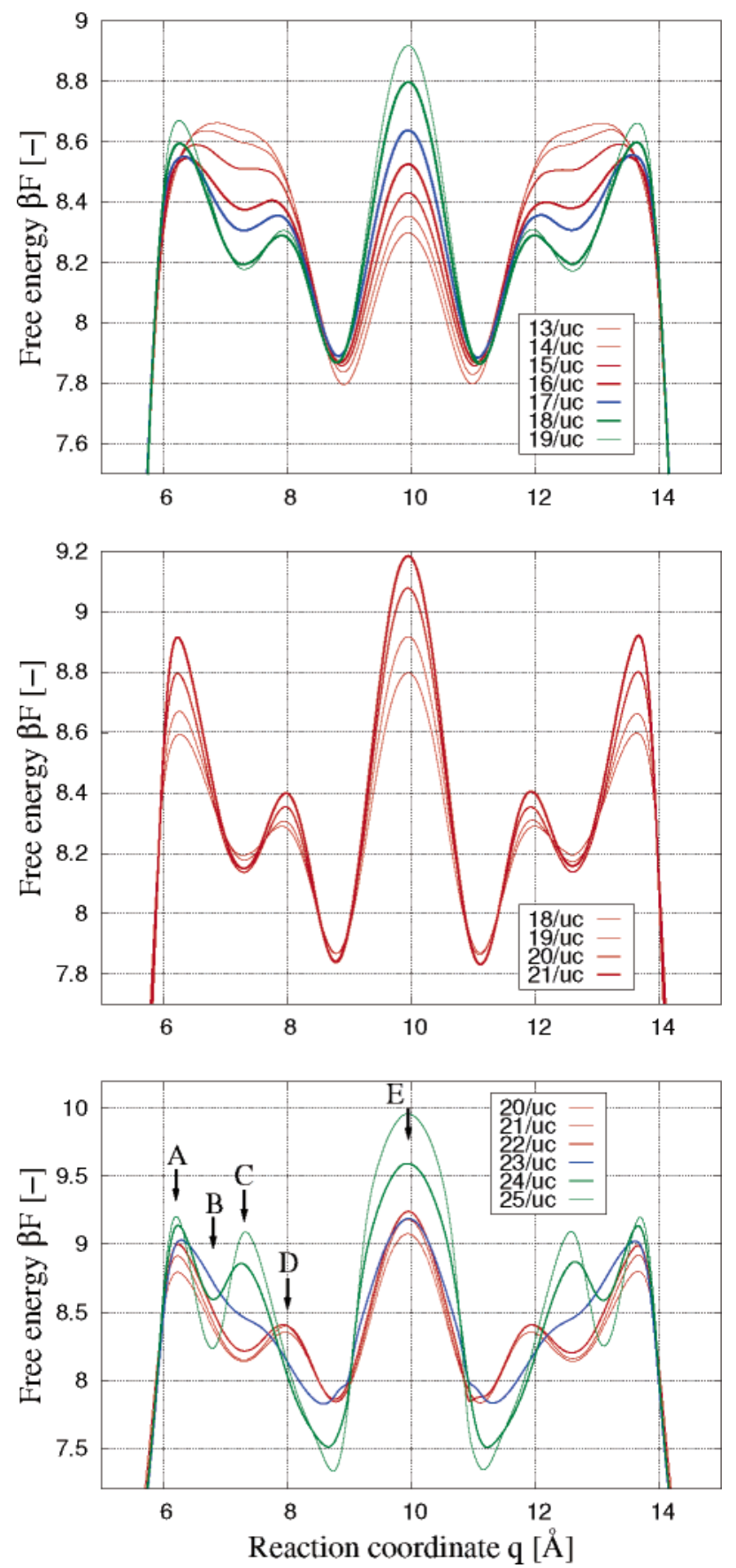

Figure 22. Selected parts from free-energy profiles, at $300 \mathrm{~K}$, of methane in the MFI-type zeolite mapped along the $y$ direction, at loadings of 13 to 19 (top), 18 to 21 (middle), and 20 to 25 (bottom) molecules per unit cell. Loadings that correspond to a peak in the diffusion are displayed in blue, lower loadings in red, and higher loadings in green.

is the sharp increase of the peak at D, causing a fast decrease of the $D_{\mathrm{c}, y}$ value as a function of loading (see Figure 22 (middle)), until it reaches zero at the maximum loading, which is about 30 molecules per unit cell. The decrease is only interrupted by a small peak in $D_{\mathrm{c}, y}$ at 23 molecules per unit cell. At a loading between 22 and 25 molecules per unit cell, the free-energy profiles change significantly (see Figure 22 (bottom)): the peaks at $\mathrm{A}$ and $\mathrm{E}$ increase, the peak at $\mathrm{D}$ vanishes, and a new peak appears at $\mathrm{C}$. This change signifies a transition from four to eight adsorption sites per straight channel. The appearance and disappearance of peaks does not occur simultaneously. At first, the dominating effect is the increase in the peak at $\mathrm{A}$, and the diffusion continues to decrease. The sudden disappearance of the peak at $\mathrm{D}$ causes a maximum in the diffusion at 23 molecules per unit cell, which immediately vanishes at higher loadings, when the peak at $\mathrm{C}$ appears.

In a similar way, the diffusion in the $x$ direction, $D_{\mathrm{c}, x}$, can be explained. Up to a loading of about 16 molecules per unit cell, the free-energy profiles do not change much. Only the peak at $C$ slightly increases, causing a very slow decrease in the diffusion. At a loading of 16 molecules per unit cell, which corresponds to four molecules per straight channel and four molecules per zigzag channel, a reordering of the adsorbates inside the zeolite takes place. Similar to the change in the straight channels, a transition takes place from four to eight molecules per zigzag channel. This transition gives rise to a new freeenergy profile: first the free energy at B decreases, producing a small increase in the diffusion at 18-19 molecules per unit cell, followed soon after by an increase of the free energy at A and $\mathrm{C}$. This increase is so significant that the diffusion rapidly slows down until it reaches zero at the maximum loading. As is shown in Figure 21, at very high loadings, from 29 molecules per unit cell, another transition takes place. The peak at A broadens to encompass B, and simultaneously, a new peak appears at $\mathrm{D}$. This indicates that at higher-loading particles inside the straight channels are inclined to arrange in two rows (left and right of peak D).

The diffusion in the $z$ direction $\left(D_{\mathrm{c}, z}\right)$ finally, proceeds similarly to that in the $x$ direction. At low loadings, the diffusion decreases as a function of loading. At a loading of 17 molecules per unit cell, the free-energy profiles reflect the transition from four to eight molecules per zigzag channel. This transition causes a slight acceleration of the diffusion at 18-19 molecules per unit cell after which the diffusion slows down to zero. As in the $x$ direction profiles, at very high loadings, we see another transition: the peak at $\mathrm{B}$ vanishes, and new peaks appear at $\mathrm{A}$ and $\mathrm{C}$.

The total corrected diffusion of methane in MFI-type zeolite is dominated by the diffusion in the $y$ direction. It is a slowly decreasing function of loading up to about 15 molecules per unit cell. There is a maximum in the diffusion at a loading of 16-17 molecules per unit cell, after which the diffusion rapidly decreases to reach zero at the maximum loading of 32 molecules per unit cell, interrupted by a small increase in the diffusion at 23 molecules per unit cell. This is in agreement with Skoulidas and Sholl, who simulated the corrected diffusion of methane in MFI-type zeolite up to a loading of 18.5 molecules per unit cell. ${ }^{1}$

In other intersecting channel-type zeolites, such as the BEC-, ISV-, and BOG-type zeolites, we observe a very similar behavior, shown in Figure 23.

This figure shows the diffusion relative to that in the infinite dilution limit. The diffusion behavior of methane in BOG-type zeolite is almost identical to that in MFI. The maximum loading of the two zeolites is similar and the free-energy profiles (and thus the particle distributions) show a similar loading dependence. However, the diffusion in BOG-type zeolite does not have maxima at 17 and 23 molecules per unit cell. The diffusion in BEC-type zeolite is also very similar to the other intersecting channel-type zeolites, although the maximum loading is about half that of MFI and BOG, in agreement with the relative size of the unit cell, and therefore the diffusion behavior is shifted to lower loadings. The loading dependence in ISV-type zeolite is, for the most part, in agreement with that in the other intersecting channel-type zeolites. However, the low-loading behavior, where the diffusion slightly increases as a function 


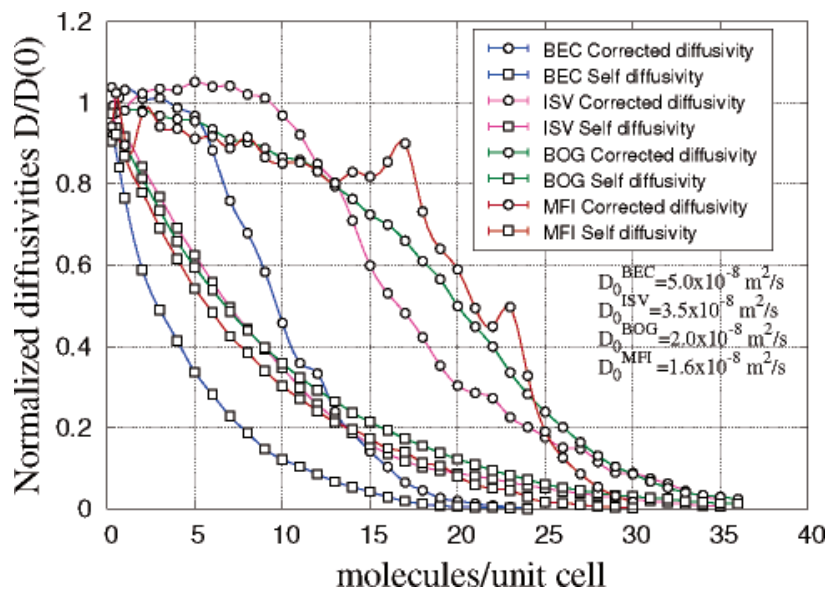

Figure 23. Relative self and corrected diffusivity of methane in intersecting channel-type zeolites BEC, ISV, BOG and MFI as a function of loading at $300 \mathrm{~K}$.

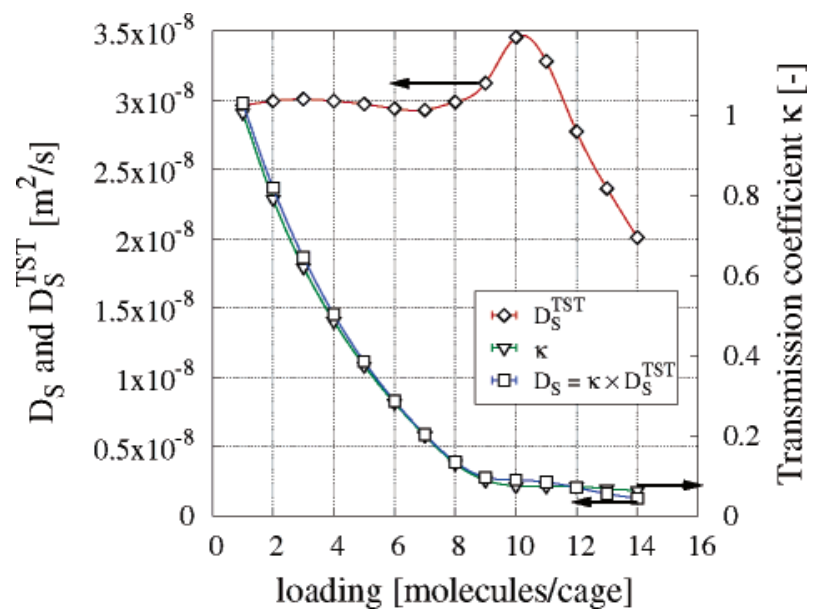

Figure 24. Diffusion of methane in FAU-type zeolite as a function of loading and the two contributions to this diffusion: the free-energy part, given by the transition state self-diffusion coefficient, and the collision part, given by the transmission coefficient.

of loading, is slightly different and could even be considered slightly cagelike.

In summary, the trends in the behavior of the corrected diffusion as a function of loading are very similar for all studied types of intersecting-channel type zeolites: at low loading, it is a slowly diminishing function of loading, and at intermediate loadings, it decreases faster, until the diffusion comes to a halt at the maximum loading of the structure. There are small deviations from this behavior, small peaks and valleys, and these can be accurately explained in the same manner as shown for MFI.

C. Diffusion in FAU-Type Zeolite. In the discussion of the diffusion of methane in cage-type zeolites, FAU-type zeolite clearly differed from other cage-type zeolites such as ERI, CHA, and LTA, in the behavior of both the self and the corrected diffusion. Whereas the diffusion of the other cage-type zeolites exhibits a maximum, for FAU, both $D_{\mathrm{s}}$ and $D_{\mathrm{c}}$ are a decreasing function of loading (see Figures 10 and 17). The self diffusion seems to behave in a way similar to that in the channel- and intersecting channel-type systems, while the corrected diffusion is almost linear, up to a loading of about 9 molecules per cage. In Figure 24, we split the self diffusion in the two contributions, $D^{\mathrm{TST}}$ and $\kappa$. The free-energy profiles from which the values of $D^{\mathrm{TST}}$ were calculated are shown in Figure 25. These profiles were obtained by choosing one of the body diagonals of the

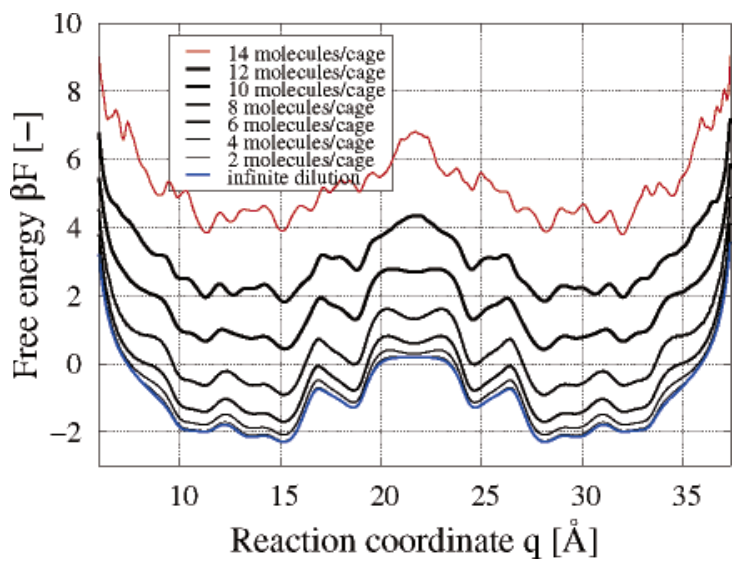

Figure 25. Free-energy profiles for methane in FAU at $300 \mathrm{~K}$, mapped on the body diagonal $(1,1,1)$.

cubic unit cell as the reaction coordinate: the body diagonal from coordinate $(0,0,0)$ to $(1,1,1)$. In this way, the reaction coordinate passes through two FAU cages, and crosses the window dividing the two perpendicularly. Figure 24 proves that this is a perfect reaction coordinate because the value of $\kappa$ at zero loading is 1 .

Surprisingly, while the free energy of the system does change when the loading is increased, the value of $D^{\mathrm{TST}}$ remains approximately constant, up to a loading of 8 molecules per cage (see Figure 25). It appears that, while the value of the free energy increases with loading, this change is more or less uniform over the entire reaction coordinate, resulting in a constant value of $D^{\mathrm{TST}}$. Therefore, the behavior of the self diffusion is completely determined by $\kappa$. This can be explained by the fact that FAUtype zeolite not only consists of very large cages but also its windows are very large $\left(R_{\mathrm{wtc}}=1.22\right)$, and up to intermediate loadings, the zeolite does not form a very strong confinement. Only at loadings of higher than 8 molecules per unit cell, we see a maximum in the diffusion. By now, the zeolite has filled up enough for the particles to experience the confinement as a cage/window-type system and exhibit the associated diffusion behavior. However, at these loadings, the diffusion is very low in comparison to that at the infinite dilution limit and the cagetype behavior is hardly distinguishable.

The corrected diffusion at low loadings is equally unaffected by free-energy differences and follows approximately $(1-\theta)$ behavior: a linear decrease of the corrected diffusion coefficient as a function of loading, as we would expect for particles performing a random walk on a lattice. Again, at loadings higher than 8 molecules per cage, the corrected diffusion coefficient increases, in accordance with $D^{\mathrm{TST}}$, and goes through a maximum at about 11 molecules per cage. We can conclude that FAU-type zeolite forms a very weak confinement up to a loading of 8 molecules per cage. At higher loadings, it can be regarded as a cage-type zeolite.

\section{Discussion}

In the previous sections, we have divided the zeolites in four groups, that all have their own specific diffusion behavior. In this section, we will compare the results with those predicted by the Reed-Ehrlich model. We calculated adsorption isotherms at $300 \mathrm{~K}$ for each of the zeolite types and obtained $1 / \gamma$ by computing the derivative of the isotherm with respect to the fugacity, as a function of the loading

$$
\left(\frac{1}{\gamma}\right)(q)=\left(\frac{\partial \ln q}{\partial \ln f}\right)(q)
$$



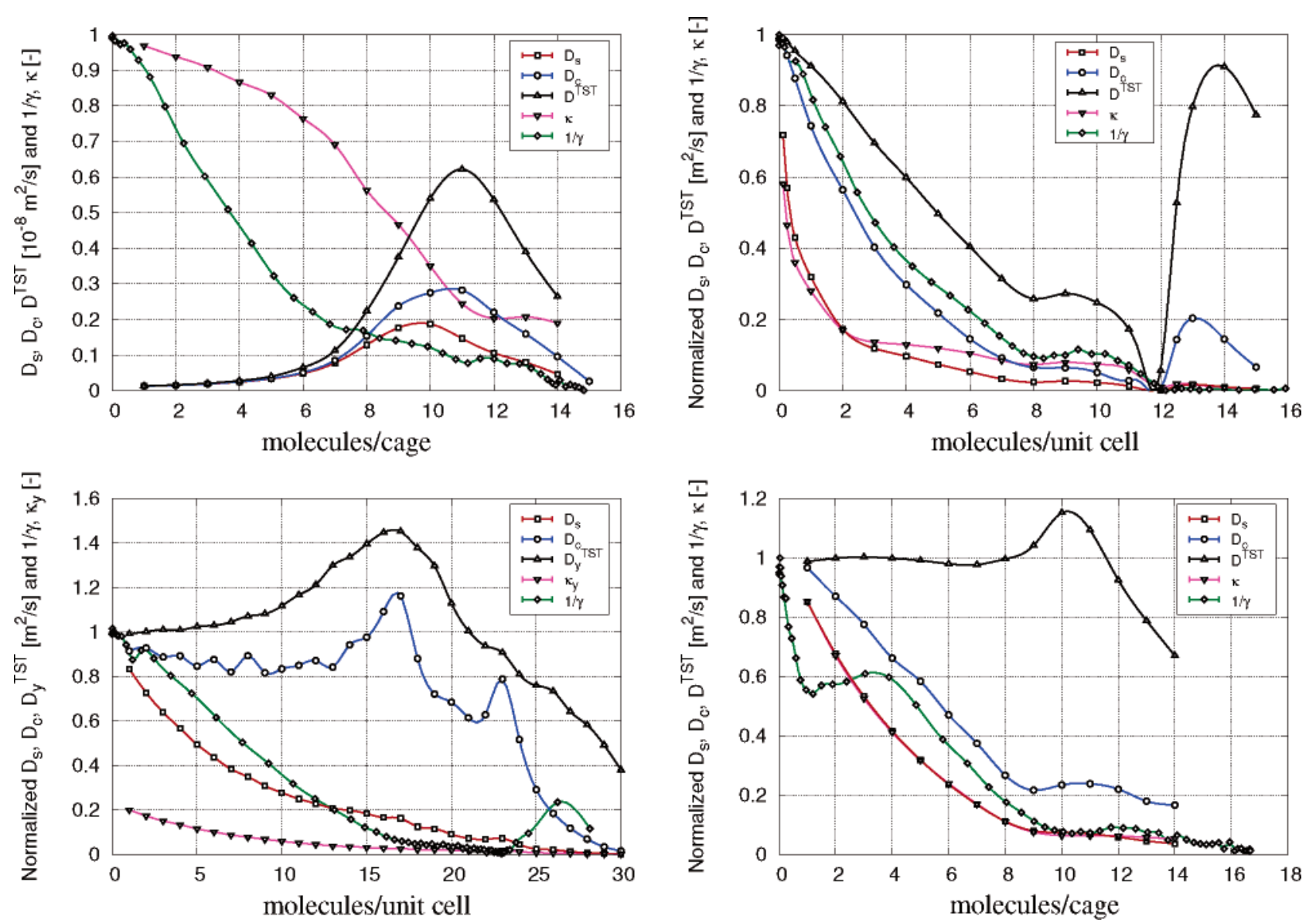

Figure 26. Compiled data for one representative zeolite from each of the four zeolite classes (all at $300 \mathrm{~K}$ ): cage-type zeolite LTA (top, left), channel-type zeolite AFI (top, right), intersecting channel-type zeolite MFI (bottom, left), and weak-confinement-type zeolite FAU (bottom, right). Diffusion coefficients, $D_{\mathrm{s}}, D_{\mathrm{c}}, D^{\mathrm{TST}}$ (normalized for AFI-, MFI-, and FAU-type zeolites, not for LTA), $\kappa$, and $1 / \gamma$ are given. The values of $D_{\mathrm{s}}, D_{\mathrm{c}}$, $D^{\mathrm{TST}}$, and $\kappa$ for MFI are for the $y$ direction only.

If adsorbed molecules do not change the energy of neighboring sites, we expect that the free-energy profiles will not change as a function of loading, and the corrected diffusion will behave as $1 / \gamma$, as follows from eq 8 . However, if adsorbed molecules do have an effect on the energy inside the zeolite, we expect the behavior of $D_{\mathrm{c}}$ to deviate from $1 / \gamma$ behavior. The ReedEhrlich model does not predict the value of the self-diffusion coefficient as a function of loading.

Figure 26 once more shows $D_{\mathrm{s}}$ and $D_{\mathrm{c}}$ as a function of loading for one representative of each zeolite class, LTA, AFI, MFI, and FAU. To compare the diffusion behavior at finite loading at that at the infinite dilution limit, the plotted diffusion coefficients have been normalized with respect to the diffusion at zero loading, except for LTA. The two components of the self diffusion, $D^{\mathrm{TST}}$ (also normalized with respect to $D_{0}^{\mathrm{TST}}$ ) and $\kappa$, and the Reed-Ehrlich prediction for the diffusion, $1 / \gamma$, are also plotted. The diffusion coefficients shown for MFI-type zeolite are for the $y$ direction.

In LTA-type zeolite, the diffusion is clearly governed by the behavior of $D^{\mathrm{TST}} ; \kappa$ only has a quantitative influence. Both the self diffusion and the corrected diffusion exhibit a maximum as a function of loading. Especially in the case of the corrected diffusion, the difference in the diffusion at the maximum with respect to the infinite dilution limit is almost 2 orders of magnitude. This sheds a new light on the order of magnitude differences found experimentally in different diffusion measurements. ${ }^{7}$ Macroscopic methods typically measure collective diffusivities (corrected and transport diffusion coefficients), while microscopic methods measure self-diffusion coefficients.
When the loading dependence of both types of diffusion is taken into consideration, it is not surprising that large deviations occur between different measurements.

It is clear that for cage-type zeolites, such as LTA, the ReedEhrlich method requires modification. The adsorbed molecules have a strong influence on the energy of neighboring adsorption sites, and the free-energy barriers change significantly when the loading is increased, thereby increasing the values of $D^{\mathrm{TST}}$. The correlations in a high-barrier system, such as LTA, contained in $\kappa$ are relatively low, and furthermore, their decrease is slower than the rapid increase in $D^{\mathrm{TST}}$. The result is that both $D_{\mathrm{s}}$ and $D_{\text {c }}$ show a qualitative behavior similar to that of $D^{\text {TST }}$ and very much unlike the behavior expected from the Reed-Ehrlich model.

In AFI-type zeolite, the behavior is altogether different. $D_{\mathrm{s}}$, $D_{\text {c }}$, and $D^{\mathrm{TST}}$ are all decreasing functions of loading. Up to a loading of 8 molecules per unit cell, $D^{\text {TST }}$ decreases approximately linearly, and $\kappa$ is a rapidly decreasing function of loading. Although the adsorbed molecules do change the energy of their environment, and thus $D^{\mathrm{TST}}$, the behavior of $D_{\mathrm{s}}$ is governed by $\kappa$ : the higher the loading, the more interparticle collisions occur, and these are the limiting factor for the diffusion in smooth channel-type zeolites. When we compare the corrected diffusion as a function of loading with $1 / \gamma$, the form of the two functions is quite similar. The overlap with the Reed-Ehrlich prediction is not perfect, but it shows that the loading-dependent diffusion behavior in AFI-type zeolite is determined for a large part by the presence of other particles, by way of vacancy correlations. The adsorption isotherm for 
AFI has a near-Langmuir form up to a loading of about 8 molecules per unit cell. At this loading there is a clear inflection. A second inflection is visible at 12 molecules per unit cell. Both inflections are reflected in the value of $1 / \gamma$, and even in $D^{\text {TST }}$ : a reordering of the molecules results in a new form of the freeenergy profile. $1 / \gamma$ gives a reasonable estimate of $D_{\mathrm{c}}$. However, the use of the Reed-Ehrlich model to compute the exact value of $D_{\mathrm{c}}$ would require $1 / \gamma$ and additional terms describing the energetic influence of adsorbed particles.

Figure 26 shows the diffusion data for MFI in the $y$ direction only. On the basis of this figure, we can state that, because the value of $D^{\text {TST }}$ increases only slowly at low loadings, the self diffusion is, for a large part, determined by the behavior of $\kappa$.

In the corrected diffusion behavior, we can recognize the effect of the free-energy changes. While $D_{\mathrm{c}}$ does not exactly follow $D^{\text {TST }}$, the trends are similar. However, to understand the full behavior of $D_{\mathrm{c}}$, one needs to look at the separate free-energy profiles and analyze them carefully. Both $D^{\mathrm{TST}}$ and $D_{\mathrm{c}}$ suggest that something is happening at 16 molecules per unit cell, which corresponds to the loading where all 16 favorable adsorption sites (four per straight channel and four per zigzag channel) have been occupied. To increase the loading any further, new less-favorable positions have to be occupied, or the molecules have to reorganize. The latter is happening, as is visible in the free-energy profiles. Interestingly, while $1 / \gamma$ does not give a reasonable estimate of the corrected diffusion behavior, it does have a subtle bend at 16 molecules per unit cell.

We stress that MFI-type zeolite is a complicated system. While the diffusion is highest in the $y$ direction, the other two directions do have their influence on the total diffusion coefficient, and to understand the diffusion behavior, all channel directions need to be taken into consideration.

For FAU, up to a loading of about 8 molecules per unit cage, the loading-dependent diffusion behavior is completely determined by interparticle collisions. Since the free-energy profiles barely change when the loading is increased, $D_{\mathrm{s}}$ has the exactly same form as $\kappa$ and $D_{\mathrm{c}}$ exhibits the typical behavior of a particle jumping randomly on a lattice. One would expect that the $D_{\mathrm{c}}$ value in such a case would follow $1 / \gamma$ exactly, but Figure 26 makes clear that this is not the case. From four molecules per cage onward, the behavior of $D_{\mathrm{c}}$ and $1 / \gamma$ are alike, but $1 / \gamma$ goes through a large minimum at one molecule per cage, which is not reflected in $D_{\mathrm{c}}$. Why $1 / \gamma$ shows this behavior is unclear and would require additional research.

In summary, the diffusion of none of the zeolites studied here can be explained by a Reed-Ehrlich model only. In each of the cage-type, channel-type, and intersecting channel-type zeolites, adsorbed particles influence the energy of their surroundings, thus rendering the Reed-Ehrlich theory, which, in its basic form, assumes constant energy-adsorption sites, invalid. Even in the case of FAU, where the loading-dependence of the net free-energy barriers can be considered negligible, the Reed-Ehrlich model cannot be used to describe the corrected diffusion over the entire loading range.

The Reed-Ehrlich model can be adjusted to contain a term that changes as a function of the loading in the system, ${ }^{37}$ but this requires prior knowledge about the system. This term could be determined by calculating free-energy profiles, but when one has obtained these free-energy profiles, it is relatively easy and quick to compute a diffusion coefficient from them using dcTST instead of the Reed-Ehrlich model. However, the Reed-Ehrlich model could perhaps be used to estimate the height of freeenergy barriers by using it in combination with MD.

Another remarkable observation can be made from the

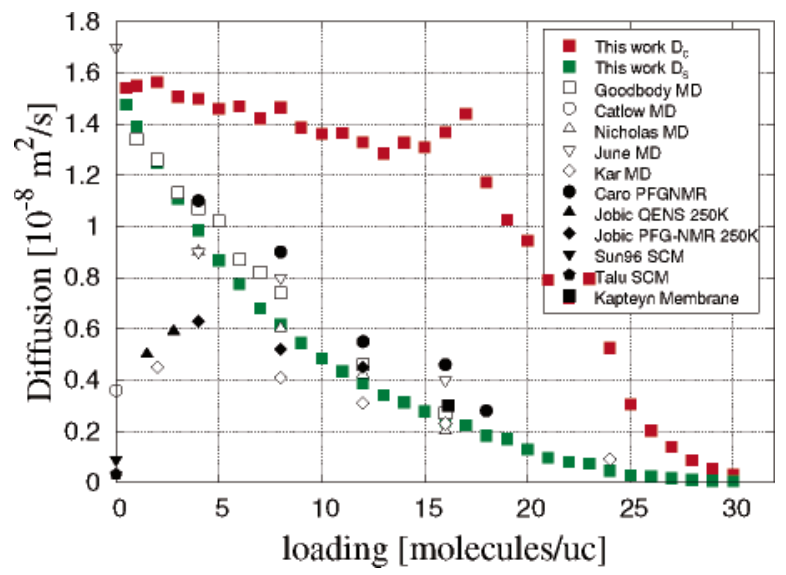

Figure 27. Simulation results for the diffusion of methane in MFItype silica as a function of the adsorbate loading, together with results obtained by several other groups, through simulation ${ }^{13-17}$ and experiment. ${ }^{29-31,93,94}$ Data were obtained at $300 \mathrm{~K}$, except for the results of Jobic et al., which were obtained at $250 \mathrm{~K}$.

comparison of the four zeolite groups. Whereas the specific loading-dependence of the diffusion is different in each group, we observe that neither the self diffusion nor the corrected diffusion is ever constant over the entire loading range. In every zeolite topology, we can assign regimes where the diffusion increases or decreases. At high loadings, it is imperative that both $D_{\mathrm{s}}$ and $D_{\mathrm{c}}$ approach zero because of the packing effects that halt the diffusion, regardless of the type of zeolite. The loading at which the final decrease sets in is determined by the zeolite type, topology, and size. Even in MFI-type zeolite, where $D_{\text {c }}$ was hitherto believed to be constant, ${ }^{1}$ the diffusion eventually goes down to zero. This implies that the Darken approximation, which states that the corrected diffusion can be assumed to be constant over loading, generally is not valid, outside of a small range near the infinite dilution limit where $D_{\mathrm{s}}$ and $D_{\mathrm{c}}$ are equal.

\section{Comparison with Experimental Data}

To validate our method, we compare our simulation results with experimental results for the two most commonly used zeolites: MFI and FAU.

Figure 27 shows our simulation results for the diffusion of methane in MFI-type silica as a function of the adsorbate loading, ${ }^{33}$ together with results obtained by several other groups, through simulation ${ }^{13-17}$ and experiment. ${ }^{29-31,93,94}$ The experimental data (black symbols) are self-diffusion coefficients, except those obtained by single-crystal membrane (SCM) measurements, which should be corrected for transport diffusivities. The results have been plotted against the loading as reported in the original papers, wherever possible. Unspecified loadings have been estimated from the reported pressures with the aid of a calculated adsorption isotherm.

Although some results show a marked deviation, both singlecrystal membrane studies, ${ }^{93,94}$ carried out at the zero-loading limit, yielded a diffusion that is much slower than that found by other methods, the overall correlation between different experimental and simulation results is good. We can conclude that the methane force field of ref 50 predicts the diffusion coefficient accurately.

The deviation observed between the single-crystal studies and most other studies is probably the result of the existence of both internal and external diffusional barriers. The more macroscopic a measurement method is, the larger the influence of the internal barriers. QENS is the experimental technique which is least affected by the existence of internal barriers. ${ }^{95,96}$ As the internal 
barriers are relatively small for methane in MFI, it is expected that the diffusivities obtained by macroscopic and microscopic methods will be further apart for longer alkanes.

As faujasite (FAU) is an important zeolite in practical applications, many experimental and simulation studies have been published. Most of these studies focus on the diffusion of aromatics, ${ }^{97-101}$ but some groups have also considered hydrocarbons. $^{102-108}$

It is interesting to compare our results with the simulations of Chempath et al. ${ }^{103}$ The molecular dynamics simulations of Chempath et al. were interpreted assuming a linear decrease of the MS diffusion coefficient as a function of loading. ${ }^{37}$ At a fractional loading of 0.8 , a single simulation point was reported that deviated from this line. Comparison with our results show that this deviation is significant.

For benzene in FAU, Auerbach and co-workers ${ }^{101}$ and Snyder et al. ${ }^{109}$ found in their kinetic Monte Carlo simulations an increase of the corrected diffusion coefficient at high loading, which was attributed to the repulsive adsorbate-adsorbate interactions. We can add now that, as benzene is a much larger molecule than methane, the adsorbate experiences the cages of Y much more like cages, and hence this system behaves much more like a cage-type zeolite than methane. For hexane and 2-methyl pentane, Van Baten and Krishna ${ }^{110}$ observed a behavior similar to that of methane. It would be interesting to investigate the behavior for longer chains, as one could argue that for the longer alkanes, the window of FAU might be an entropic barrier, and this would show cagelike behavior again.

\section{Conclusion}

We have compared the loading-dependent behavior of the self diffusion and corrected diffusion for methane in twelve different zeolite topologies. On the basis of their characteristics, we can divide these twelve topologies into four zeolite groups. Each of the four zeolite groups shows very distinct diffusion behavior as a function of loading.

In cage-type zeolites, we observe a maximum in both the self and corrected diffusion caused by decreased free-energy barriers, after which both slow to zero. Diffusion behavior in channel-type zeolites is highly dependent on the ratio between the narrowest and widest part of the channels, $R_{\mathrm{ctw}}$. In the smoothest channels, both $D_{\mathrm{s}}$ and $D_{\mathrm{c}}$ are a steeply decreasing function of loading. In the most cagelike channels, those with the highest value of $R_{\mathrm{ctw}}, D_{\mathrm{s}}$, and $D_{\mathrm{c}}$ behave as true cage-type systems. The zeolites with an intermediate value of $R_{\mathrm{ctw}}$ behave in an intermediate way. Generally, $D_{\mathrm{s}}$ is still monotonically decreasing, although the curve is less steep than in the smoothest channels. Dependent on the exact value of $R_{\mathrm{ctw}}, D_{\mathrm{c}}$ can have a slight cagelike behavior, resulting in a small maximum at low loadings. In intersecting channel-type zeolites, $D_{\mathrm{s}}$ generally behaves similar to that in channel-type zeolites of intermediate smoothness. $D_{\mathrm{c}}$ has two consecutive diffusion regimes: ${ }^{111}$ first a slow linear decrease, until at least one of the channels has reached its maximal loading, and then a sharper plunge to reach zero at the saturation loading of the zeolite.

In any zeolite type, at high loadings, both $D_{\mathrm{s}}$ and $D_{\mathrm{c}}$ drop to zero. This observation implies that the Darken approximation cannot be used outside a small region near zero loading. We note that in simulations one can apply pressures that are higher than those used in experiments, where full saturation is often defined as the loading at which there is an equilibrium with a surrounding liquid phase. The maximum loading in simulations is often higher than this full-saturation capacity. Experimentally is has been shown that the self diffusion can slow more than 2 orders of magnitude at these higher loadings (see, for example, Kärger et al. ${ }^{112}$ for $n$-alkanes in $\mathrm{NaX}$ ), but this diffusion was still nonzero.

We stress that the ordering of molecular sieve structures in classes depends strictly on the combination of adsorbate and adsorbent. For example, a cage that appears very large for methane molecules can in fact be a very tight confinement for benzene. When applying this classification to larger molecules, sieve structures can therefore switch class, but the general behavior will be the same: when the cages are large (with respect to the adsorbed molecule) and the windows are narrow, the diffusion as a function of loading will go through a maximum; when the confinement is experienced as a smooth channel, the diffusion is a decreasing function of loading (this has also been observed for small alkanes in carbon-nanotubes ${ }^{113}$ ); and when the confinement consists of intersecting channels, $D_{S}$ will be monotonically decreasing as a function of loading, and $D_{C}$ will show a kink. The method employed in this study can be used to make a classification of pore structures for any given adsorbate molecule.

Acknowledgment. This work is supported by the Deutsche Forschungsgemeinschaft (DFG, priority program SPP 1155), the EC though the Marie Curie EXT project MEXT-CT-2005023311, and The Netherlands Research Council (CW). We thank R. Krishna and H. Jobic for stimulating discussions, and NWO/ NCF for computational resources.

\section{References and Notes} 10141

(1) Skoulidas, A. I.; Sholl, D. S. J. Phys. Chem. A 2003, 107, 10132-

(2) Fritzsche, S.; Gaub, M.; Haberlandt, R.; Hofmann, G. J. Mol. Model. 1996, 2, 286-292.

(3) Snurr, R. Q.; Bell, A. T.; Theodorou, D. N. J. Phys. Chem. 1994 98, 11948-11961.

(4) Auerbach, S. M. Int. Rev. Phys. Chem. 2000, 19, 155-198.

(5) Ramanan, H.; Auerbach, S. M. In Fluid Transport in Nanopores; Fraissard, J., Conner, W. C., Eds.; NATO-ASI Series C; Kluwer Academic Publishers: Dordrecht, The Netherlands, 2004. 4263.

(6) Jobic, H.; Karger, J.; Bee, M. Phys. Rev. Lett. 1999, 82, 4260-

(7) Kaerger, J.; Ruthven, D. M. Diffusion in Zeolites and Other Microporous Solids; John Wiley \& Sons: New York, 1992.

(8) Maginn, E. J.; Bell, A. T.; Theodorou, D. N. J. Phys. Chem. 1996, $100,7155-7173$.

(9) Krishna, R.; Baten, J. M.; van Dubbeldam, D. J. Phys. Chem. B 2004, 108, 14820.

(10) Xiao, J.; Wei, J. Chem. Eng. Sci. 1992, 47, 1123.

(11) Fritzche, S.; Wolfsberg, M.; Haberlandt, R.; Demontis, P.; Suffritti, G. B.; Tilocca, A. Chem. Phys. Lett. 1998, 296, 253.

(12) Saravanan, C.; Jousse, F.; Auerbach, S. M. Phys. Rev. Lett. 1998 $80,5754-5757$.

(13) Goodbody, S. J.; Watanabe, J. K.; Gowan, D. M.; Walton, J. P. R. B.; Quirke, N. J. Chem. Soc., Faraday Trans. 1991, 87, 1951.

(14) Catlow, C. R. A.; Freeman, C. M.; Vessal, B.; Tomlinson, S. M.; Leslie, M. J. Chem. Soc., Faraday Trans. 1991, 87, 1947.

(15) Nicholas, J. B.; Trouw, F. R.; Mertz, J. E.; Iton, L. E.; Hopfinger, A. J. J. Phys. Chem. 1993, 97, 4149.

(16) Kar, S.; Chakravarty, C. J. Phys. Chem. A 2001, 105, 5785-5793.

(17) June, R. L.; Bell, A. T.; Theodorou, D. N. J. Phys. Chem. 1990, $94,8232-8240$ 9474.

(18) Tepper, H. L.; Briels, W. J. J. Chem. Phys. 2002, 116, 9464-

(19) Schüring, A.; Auerbach, S. M.; Fritzsche, S.; Haberlandt, R. J. Chem. Phys. 2002, 116, 10890-10894.

(20) Jousse, F.; Auerbach, S. M. J. Chem. Phys. 1997, 107, 9629-9639.

(21) Tunca, C.; Ford, D. M. J. Chem. Phys. 1999, 111, 2751-2760.

(22) Tunca, C.; Ford, D. M. J. Phys. Chem. B 2002, 106, 10982-10990.

(23) Tunca, C.; Ford, D. M. Chem. Eng. Sci. 2003, 58, 3373-3383.

(24) Ghorai, P. Kr.; Yashonath, S. J. Chem. Phys. 2004, 120, 53155321.

(25) Heink, W.; Kärger, J.; Pfeifer, H.; Salverda, P.; Datema, K. P.; Nowak, A. J. Chem. Soc., Faraday Trans. 1992, 88, 515-519. 
(26) June, R. L.; Bell, A. T.; Theodorou, D. N. J. Phys. Chem. 1991, 95, 8866-8878.

(27) Dubbeldam, D.; Calero, S.; Maesen, T. L. M.; Smit, B. Phys. Rev. Lett. 2003, 90, 245901.

(28) Dubbeldam, D.; Calero, S.; Maesen, T. L. M.; Smit, B. Angew. Chem., Int. Ed. 2003, 42, 3624-3626.

(29) Caro, J.; Bulow, M.; Schirmer, W.; Karger, J.; Heink, W.; Pfeifer, H. J. Chem. Soc., Faraday Trans. 1985, 81, 2541.

(30) Jobic, H.; Bee, M.; Caro, J.; Bulow, M.; Karger, J. J. Chem. Soc., Faraday Trans. 1989, 85, 4201.

(31) Kapteyn, F.; Bakker, W. J. W.; Zheng, G.; Moulijn, J. A. Chem. Eng. J. 1995, 57, 145-153.

(32) Beerdsen, E.; Smit, B.; Dubbeldam, D. Phys. Rev. Lett. 2004, 93 248301.

(33) Beerdsen, E.; Dubbeldam, D.; Smit, B. Phys. Rev. Lett. 2005, 95, 164505 .

(34) Beerdsen, E.; Dubbeldam, D.; Smit, B. Phys. Rev. Lett. 2006, 96 , 044501 .

(35) Dubbeldam, D.; Beerdsen, E.; Vlugt, T. J. H.; Smit, B. J. Chem. Phys. 2005, 122, 224712.

(36) Reed, D. A.; Ehrlich, G. Surf. Sci. 1981, 102, 588-609.

(37) Krishna, R.; Paschek, D.; Baur, R. Microporous Mesoporous Mater. 2004, 76, 233-246.

(38) Papadopoulos, G. K.; Jobic, H.; Papadopoulos, D. N. J. Phys. Chem. B 2004, 108, 12748-12756. 549 .

(39) Krishna, R.; van Baten, J. M. Chem. Phys. Lett. 2006, 420, 545-

(40) Krishna, R.; van Baten, J. M. Ind. Eng. Chem. Res. 2006, 45, 20842093.

(41) Krishna, R.; Paschek, D. Chem. Phys. Lett. 2001, 33, 278-284.

(42) Krishna, R.; Smit, B.; Calero, S. Chem. Soc. Rev. 2002, 31, 185194.

(43) Vlugt, T. J. H.; Krishna, R.; Smit, B. J. Phys. Chem. B 1999, 103, $1102-1118$

(44) Gorring, R. L. J. Catal. 1973, 31, 13-26.

(45) Nitsche, J. M.; Wei, J. AIChE. J. 1991, 37, 661-670.

(46) Runnebaum, R. C.; Maginn, E. J. J. Phys. Chem. B 1997, 101, 6394-6408.

(47) Talu, O.; Sun, M. S.; Shah, D. B. AIChE. J. 1998, 44, 681-694.

(48) Bezus, A. G.; Kiselev, A. V.; Lopatkin, A. A.; Du, P. Q. J. J. Chem.

Soc., Faraday Trans. 2 1978, 74, 367-379.

(49) Ryckaert, J. P.; Bellemans, A. Faraday Dicuss. 1978, 66, 95-106. (50) Dubbeldam, D.; Calero, S.; Vlugt, T. J. H.; Krishna, R.; Maesen,

T. L. M.; Beerdsen, E.; Smit, B. Phys. Rev. Lett. 2004, 93, 088302-1.

(51) Dubbeldam, D.; Calero, S.; Vlugt, T. J. H.; Krishna, R.; Maesen, T. L. M.; Smit, B. J. Phys. Chem. B 2004, 108, 12301-12313.

(52) Bennett, C. H. In Diffusion in Solids: Recent Developments; Nowick, A. S., Burton, J. J., Eds.; Academic Press; New York, 1975; pp $73-113$.

(53) Chandler, D. J. Chem. Phys. 1978, 68, 2959.

(54) Frenkel, D.; Smit, B. Understanding Molecular Simulation, 2nd ed.; Academic Press: London, U.K., 2002.

(55) Auerbach, S. M. In Fluid Transport in Nanopores; Fraissard, J., Conner, W. C., Eds.; NATO-ASI Series C; Kluwer Academic Publishers; Dordrecht, The Netherlands, 2004.

(56) Maesen, T. L. M.; Schenk, M.; Vlugt, T. J. H.; de Jonge, J. P.; Smit, B. J. Catal. 1999, 188, 403-412.

(57) http://www.iza-structure.org/databases/.

(58) Pluth, J. J.; Smith, J. V. J. Am. Chem. Soc. 1980, 102, 47044708.

(59) Gard, J. A.; Tait, J. M. In Proceedings of the Third International Conference on Molecular Sieves, Recent Progress Reports; Uytterhoven, J. B., Ed.; Leuven University Press: Zurich, Switzerland, 1973; pp 9499. 208

(60) Calligaris, M.; Nardin, G.; Randaccio, L. Zeolites 1986, 3, 205-

(61) Hriljac, J. J.; Eddy, M. M.; Cheetham, A. K.; Donohue, J. A. J. Solid State Chem. 1993, 106, 66-72.

(62) Qiu, S.; Pang, W.; Kessler, H.; Guth, J. L. Zeolites 1989, 9, 440444.

(63) Fyfe, C. A.; Gies, H.; Kokotailo, G. T.; Marler, B.; Cox, D. E. J. Phys. Chem. 1990, 94, 3718-3721.

(64) Barrer, R. M.; Villiger, H. Z. Kristallogr. 1969, 128, 352-270.

(65) Patinec, V.; Wright, P. A.; Lightfoot, P.; Aitken, R. A.; Cox, P. A.

J. Chem. Soc., Dalton Trans. 1999, 3909-3911.

(66) van Koningsveld, H.; van Bekkum, H.; Jansen, J. C. Acta Crystallogr. 1987, B43, 127-132.

(67) Pluth, J. J.; Smith, J. V. Am. Mineral. 1990, 75, 501-507.

(68) Liu, Z.; Ohsuna, T.; Terasaki, O.; Camblor, M. A.; Diaz-Cabanas,

M.; Hiraga, K. J. Am. Chem. Soc. 2001, 123, 5370-5371.

(69) Villaescusa, L. A.; Barrett, P. A.; Camblor, M. A. Angew. Chem., Int. Ed. 1999, 38, 1997-2000.
(70) Maesen, T. L. M.; Beerdsen, E.; Calero, S.; Dubbeldam, D.; Smit, B. J. Catal. 2006, 237, 278-290.

(71) Ribeiro, F. R.; Rodriguez, A.; Rollmann, D.; Naccache, C. In Zeolites: Science and Technology; Ribeiro, F. R., Ed.; Martinus Nijhoff Publishers: The Hague, The Netherlands, 1984.

(72) Calero, S.; Schenk, M.; Dubbeldam, D.; Maesen, T. L. M.; Smit,

B. J. Catal. 2004, 228, 121-129.

(73) Bhatia, S. Zeolite Catalysis: Principles and Applications; CRC Press Inc.: Boca Raton, FL, 1990.

(74) Gupta, V.; Nivarthi, S. S.; Keffer, D.; McCormick, A. V.; Davis, H. T. Science 1996, 274, 164-164.

(75) Gupta, V.; Nivarthi, S. S.; McCormick, A. V.; Davis, H. T. Chem. Phys. Lett. 1995, 247, 596-600.

(76) Hahn, K.; Kärger, J. J. Phys. Chem. B 1998, 102, 5766-5771.

(77) Jobic, H.; Hahn, K.; Karger, J.; Bee, M.; Tuel, A.; Noack, M.; Girnus, I.; Kearley, G. J. J. Phys. Chem. B 1997, 101, 5834-5841.

(78) Demontis, P.; Gonzalez, J. G.; Suffritti, G. B.; Tilocca, A. J. Am Chem. Soc. 2001, 123, 5069-5074.

(79) Tepper, H. L.; Hoogenboom, J. P.; van der Vegt, N. F. A.; Briels, W. J. J. Chem. Phys. 1999, 110, 11511-11516. 8597.

(81) Nelson, P. H.; Auerbach, S. M. Chem. Eng. J. 1999, 74, 43-56. (82) Nelson, P. H.; Auerbach, S. M. J. Chem. Phys. 1999, 110, 92359243.

(83) Sholl, D. S.; Fichthorn, K. A. J. Chem. Phys. 1997, 107, 43844389 .

(84) Dume, C.; Holderick, W. F. Appl. Catal., A 1999, 167, 183-193.

(85) Schenk, M.; Calero, S.; Maesen, T. L. M.; van Benthem, L.;

Verbeek, M. G.; Smit, B. Angew. Chem., Int. Ed. 2002, 41, 2500-2502.

(86) Davis, M. E. Nature (London) 2002, 417, 813-821.

(87) Zhang, W.; Smirniotis, P. G. Catal. Lett. 1999, 60, 223-228.

(88) Triantafillou, N. D.; Miller, J. T.; Gates, B. C. J. Catal. 1995, 155 $131-140$ 5793.

(89) Demontis, P.; Suffritti, G. B. J. Phys. Chem. B 1997, 101, 5789-

(90) Schüring, A.; Auerbach, S. M.; Fritzsche, S.; Haberlandt, R. J. Chem. Phys. 2002, 116, 10890-10894.

(91) Maris, T.; Vlugt, T. J. H.; Smit, B. J. Phys. Chem. B 1998, 102, $7183-7189$

(92) Pascual, P.; Ungerer, P.; Tavitian, B.; Pernot, P.; Boutin, A. Phys. Chem. Chem. Phys. 2003, 5, 3684-3693.

(93) Sun, M. S.; Talu, O.; Shah, D. B. AIChE. J. 1996, 42, 3001.

(94) Talu, O.; Sun, M. S.; Shah, D. B. AIChE. J. 1998, 44, 681-694.

(95) Vasenkov, S.; Kärger, J. Microporous Mesoporous Mater. 2002 $55,139-145$.

(96) Vasenkov, S.; Böhlmann, W.; Galvosas, P.; Geier, O.; Liu, H.; Kärger, J. J. Phys. Chem. B 2001, 105, 5922-5927.

(97) Mosell, T.; Schrimpf, G.; Brickmann, J. J. Phys. Chem. B 1997, $101,9476-9484$

(98) Mosell, T.; Schrimpf, G.; Brickmann, J. J. Phys. Chem. B 1997, 101, 9485-9494.

(99) Saravanan, C.; Auerbach, S. M. J. Chem. Phys. 1997, 107, 81208131.

(100) Saravanan, C.; Auerbach, S. M. J. Chem. Phys. 1997, 107, 81328137.

(101) Saravanan, C.; Jousse, F.; Auerbach, S. M. J. Chem. Phys. 1998 $108,2162-2169$

(102) Bandyopadhyay, S.; Yashonath, S. Chem. Phys. Lett. 1994, 223, $363-368$.

(103) Chempath, S.; Krishna, R.; Snurr, R. Q. J. Phys. Chem. B 2004 108, 13481-13491.

(104) Clark, L. A.; Ye, G. T.; Gupta, A.; Hall, L. L.; Snurr, R. Q. J. Chem. Phys. 1999, 111, 1209-1222.

(105) Sanborn, M. J.; Snurr, R. Q. Sep. Purif. Technol. 2000, 20, 1-13.

(106) Sanborn, M. J.; Snurr, R. Q. AIChE J. 2001, 47, 2032-2041.

(107) Yashonath, S.; Demontis, P.; Klein, M. L. Chem. Phys. Lett. 1988 153, 551-555.

(108) Yashonath, S.; Thomas, J. M.; Nowak, A. K.; Cheetham, A. K. Nature 1988, 331, 601-604.

(109) Snyder, M. A.; Vlachos, D. G. J. Chem. Phys. 2005, 123, 184708.

(110) van Baten, J. M.; Krishna, R. Microporous Mesoporous Mater. 2005, 84, 179-191.

(111) Krishna, R.; Vlugt, T. J. H.; Smit, B. Chem. Eng. Sci. 1999, 54, $1751-1757$

(112) Karger, J.; Pfeifer, H.; Rauscher, M.; Walter, A. J. Chem. Soc., Faraday 1980, 76, 717-737.

(113) Skoulidas, A. I.; Ackerman, D. M.; Johnson, J. K.; Sholl, D. S. Phys. Rev. Lett. 2002, 89, 185901. 